
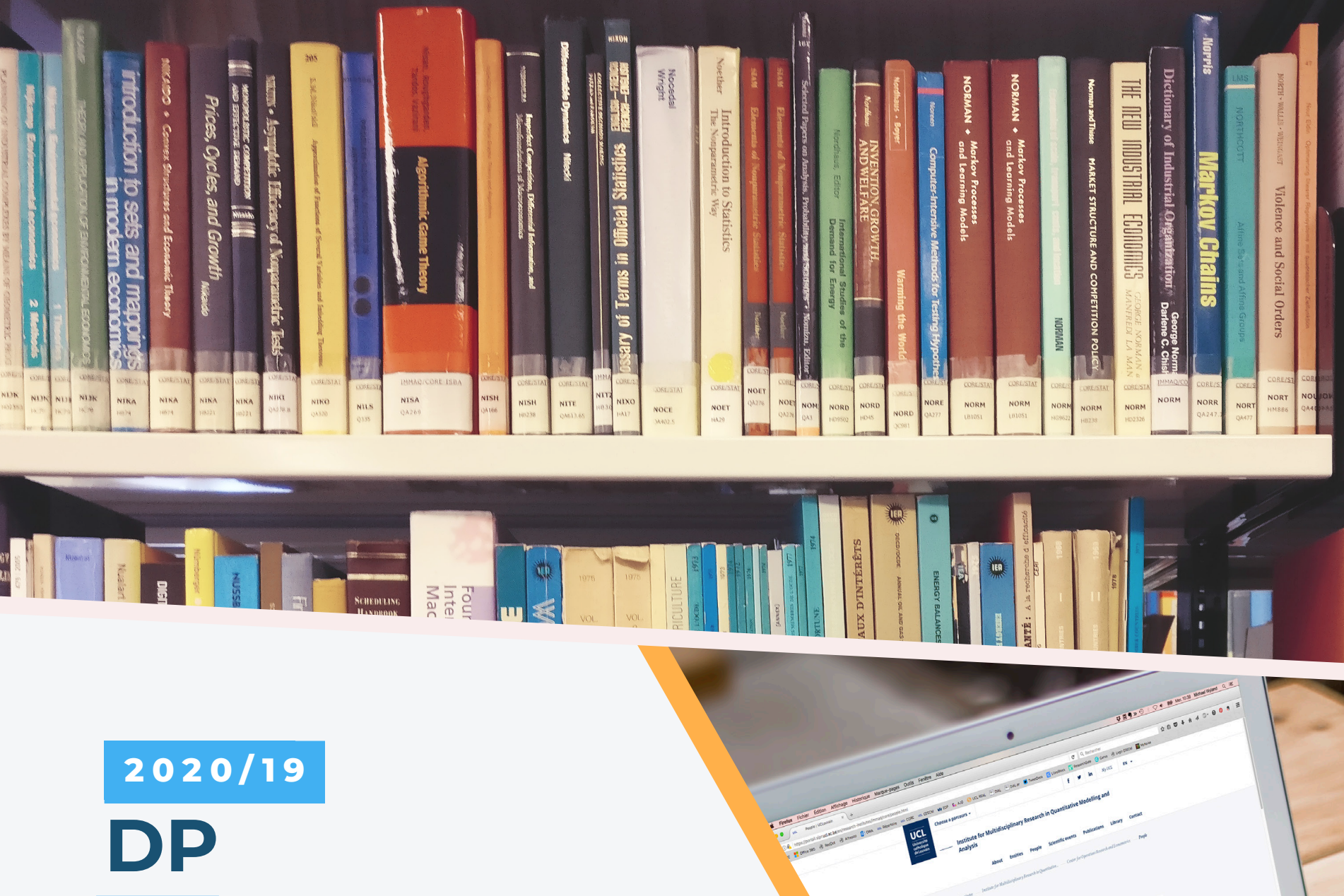

Ana Mauleon, Simon Schopohl, Akylai Taalaibekova and Vincent Vannetelbosch

Coordination on networks with farsighted and myopic agents 


\section{CORE}

Voie du Roman Pays 34, L1.03.01

B-1348 Louvain-la-Neuve

Tel (32 10) 474304

Email: immaq-library@uclouvain.be

https://uclouvain.be/en/research-institutes/

lidam/core/discussion-papers.html 


\title{
Coordination on Networks with Farsighted and Myopic Agents
}

\author{
Ana Mauleon* $\quad$ Simon Schopohl ${ }^{\dagger} \quad$ Akylai Taalaibekova ${ }^{\ddagger}$ \\ Vincent Vannetelbosch ${ }^{\S}$
}

April 29, 2020

\begin{abstract}
We study a coordination game on a fixed connected network where players have to choose between two projects. Some players are moderate (i.e. they are ex-ante indifferent between both project) while others are stubborn (i.e. they always choose the same project). Benefits for moderate players are increasing in the number of neighbors who choose the same project. In addition, players are either farsighted or myopic. Farsighted players anticipate the reactions of others while myopic players do not. We show that, when all players are farsighted, full coordination among the moderate players is reached except if there are stubborn players for both projects. When the population is mixed, the set of stable strategy profiles is a refinement of the set of Nash equilibrium strategy profiles. In fact, turning myopic players into farsighted ones eliminates little by little the inefficient Nash equilibria. Finally, we consider a social planner who can improve coordination by means of two policy instruments: adding links to the network (socialization) and/or turning myopic players into farsighted ones (education).
\end{abstract}

Key words: networks; coordination problems; stubborn players; farsighted players; stability.

JEL Classification: A14, C70, D20.

${ }^{*}$ CEREC and CORE, UCLouvain, Belgium. E-mail: ana.mauleon@usaintlouis.be

${ }^{\dagger}$ CEREC and CORE, UCLouvain, Belgium.

${ }^{\ddagger}$ CORE, UCLouvain, Belgium; CES, Université Paris 1 Panthéon-Sorbonne, France. E-mail: akylai.taalaibekova@uclouvain.be

$\S$ CORE and CEREC, UCLouvain, Belgium. E-mail: vincent.vannetelbosch@uclouvain.be 


\section{Introduction}

Coordination problems play a huge role in social and economic interactions. Partners, family and friends coordinate what to eat, how to spend the evening or where to go on vacation. Researchers coordinate with their coauthors time and date of meetings and the splitting of tasks. Firms active in research and development or joint production partnerships often need to coordinate on the choice of compatible technologies. Countries involved in trade and political alliances must agree on conventions such as language. In most of those coordination problems, agents make choices positively influenced by their neighbors (friends, collaborators, ...) in the network. ${ }^{1}$

In a network setting, we address fundamental questions about coordination games in networks. Does farsightedness solves the coordination problem? Do stubborn agents impede coordination in the presence of farsighted agents? Do we improve coordination by turning myopic agents into farsighted ones? Can a social planner reach full coordination by stimulating socialization (adding links to the network) or education (making myopic agents becoming farsighted)?

To answer those questions we study a coordination game on a fixed connected network. Players have to choose between two projects. ${ }^{2}$ Some players are moderate (i.e. they are ex-ante indifferent for both projects) while others are stubborn players (i.e. they always choose the same project independently of what others do). Benefits are supposed to be increasing in the number of friends or neighbors who choose the same project, conditionally on the project being successful. A project is said to be successful if it is adopted by enough people within the whole society. In addition of being either moderate or stubborn, each player can either be myopic or farsighted. In contrast to myopic players, a farsighted player anticipates that once she makes a choice and deviates, other players may deviate afterwards. In other words, she anticipates the influence she will have on the choices made by other players later on.

We first solve the coordination game when all players are farsighted. We propose the concept of farsightedly stable strategy profile for determining the outcome of the game. A strategy profile is farsightedly stable if there is no farsighted improving path emanating of it. ${ }^{3}$ A farsighted improving path is a sequence of strategy profiles where each time one player chooses an action based on the improvement the end strategy profile offers her relative to the current strategy profile. We show that there always exists a farsightedly stable strategy profile. Suppose there are no stubborn players or only stubborn players for a single project. Then, farsightedness leads to full coordination among the whole

\footnotetext{
${ }^{1}$ Jackson (2008) and Goyal(2007) provide a comprehensive introduction to the theory of social and economic networks. Mauleon and Vannetelbosch (2016) give an overview of the solution concepts for solving network formation games.

${ }^{2}$ Notice that all our main results hold if we would consider more than two projects.

${ }^{3}$ See Chwe (1994), Dutta, Ghosal and Ray (2005), Herings, Mauleon and Vannetelbosch (2009, 2019), Page and Wooders $(2009)$, Ray and Vohra $(2015,2019)$ among others for alternative notions of farsightedness.
} 
society, even though there is a large multiplicity of inefficient Nash equilibria. Once there are stubborn players for both projects, farsightedness does not always overcome the issue of miscoordination. Intuitively, farsighted players may fail to influence other moderate players if the only channels for influencing them go through stubborn players. Nevertheless, we provide conditions to guarantee that all moderate players fully coordinate on the same project.

We next consider the case of a mixed population consisting of myopic and farsighted players. Indeed, recent experiments provide evidence in favor of a mixed population consisting of both myopic and farsighted individuals (see Kirchsteiger, Mantovani, Mauleon and Vannetelbosch, 2016). We propose the concept of myopic-farsighted stable strategy profile for determining the outcome of the game. A strategy profile is myopic-farsighted stable if there is no myopic-farsighted improving path emanating from it. A myopicfarsighted improving path is a sequence of strategy profiles that can emerge when farsighted players choose an action based on the improvement the end strategy profile offers them relative to the current strategy profile while myopic players choose an action based on the improvement the resulting strategy profile offers them relative to the current one. We show that when all players are myopic the set of stable strategy profiles coincides with the set of Nash equilibria of the coordination game. A quite interesting result is that the set of stable profiles weakly decreases when a myopic player becomes farsighted. Hence, the set of myopic-farsighted stable strategy profiles when moderate players are both myopic and farsighted is a refinement of the set of Nash equilibrium strategy profiles. In fact, turning myopic players into farsighted ones eliminates little by little the inefficient Nash equilibria. We then look at the impact of stubbornness and at what happens for some specific networks (complete, star and line networks).

Finally, we consider a social planner who can improve coordination by means of two policy instruments: adding links to the network (socialization) and/or turning myopic players into farsighted ones (education). In the case of regular networks, especially circle networks, both instruments are substitutes when all players are moderate. However, in the presence of stubborn players, turning myopic players into farsighted ones without adding links cannot lead to full coordination among the moderate players.

One motivation for our model is the adoption of new technologies. Agents choose whether or not to adopt a new technology or keep the old one, such as in social media, where the benefit of the technology is increasing in the adoption by friends or neighbors. For being successful or profitable, the technology needs to be adopted by a certain mass of customers; otherwise, it probably disappears in the long run. In addition, we often encounter within the population agents who sticks no matter what to the old technology while others are slavishly devoted to the new technologies, like nerds. Another example is the cooperation in R\&D between researchers or firms. Clearly, researchers build a network of collaborations, where the links can be interpreted as previous cooperation on a project. The researchers can investigate into different projects. While some projects might be solved by a single unit, other projects need the cooperation of several units. 
If there are not enough units to lead the global project to a good end, they may fail to get financial support for it. The collaboration with other researchers might not give a higher payoff to the researcher directly, but could lower the effort she has to deploy for her research. While some researchers might be in principle interested in several projects, other researchers could be reluctant for starting some new projects. Other examples include the choice by the society between alternative projects for the development of the country. ${ }^{4}$

We now turn to the related literature. In our coordination game played on a fixed network, players have incentives to coordinate with their neighbors. But, there are situations where the opposite happens. Bramoullé (2007) studies anti-coordination games and defines frustration as a function of neighbors who play the same action and neighbors who choose a different action. Frustration is the highest in the complete network and the lowest in bipartite networks. Bramoullé, Lopez-Pintado, Goyal and Vega-Redondo (2004) go one-step further by endogenizing the network formation. Jackson and Watts (2002a) also look at coordination games on networks, but rather focus on repeated interactions. In each period, first a randomly selected link can be added or deleted, next a randomly chosen player can adjust her action. With a small probability a player makes a mistake which might induce other players to modify their actions later on. All players are myopic and are ex-ante indifferent between the different alternatives. For solving this game, they use the concept of stochastic stability. They find that the endogenous network not only has implications for the interaction pattern that emerges, but it also has a significant impact on the play in the coordination game relative to what would arise if the same interaction network were exogenous. Goyal and Vega-Redondo (2005) also study a coordination game which is repeatedly played on networks where players can choose between two different options. Players can revise their strategy in each period with a certain probability. In their model the links between players are paid only by one player. The authors show that depending on the costs for a link the players either coordinate on the risk-dominant action or on the efficient action.

Recently, Leister, Zenou and Zhou (2019) study a coordination game among agents on a network, who choose whether or not to take an action in an uncertain environment. Similar to our model, the value is increasing in the amount of neighbors who adopt the same action. They propose an algorithm that fully characterizes the equilibrium coordination sets within the network, and they provide an exact condition for which a single coordination set exists in the network. This condition requires that the network is balanced: the average degree of each subnetwork is not greater than the average degree of the entire network. In a related paper, Jackson and Storms (2019) analyze a game in which each player can either adopt the behavior of her neighbors or not. If a certain

\footnotetext{
${ }^{4}$ In Switzerland, people had to choose in 2018 between being candidate for organizing the Winter Olympic Games 2026 or not. The no-option reverts to allocate the proposed budget to more durable activities. The no-option won by $53,98 \%$ and from the analysis of the votes and polls it was observed that most people from the cities were voting against except those having strong ties with the ski resorts (Le Nouvelliste, 10/6/2018).
} 
amount of neighbors of a player choose to adopt some behavior, it becomes beneficial for this player to adopt this behavior as well. There can be multiple different conventions in which players in some parts of the network adopt a behavior while players in other parts of the network do not. They define communities to be groups of players who behave the same as each other in every convention.

Closest to our work, but without the network aspect is the work of Sakovics and Steiner (2012). They focus on a continuum of players where each player can either decide to invest into a project or to abstain from investment. The project has an investment threshold and is only successful if enough players invest. The players receive a noisy signal about this threshold. The authors show that depending on the noise in the signal there can be miscoordination, while if the noise is small the players coordinate their actions. ${ }^{5}$

We go further the related literature by considering the impact of a mixed population along two dimensions (moderate versus stubborn players, farsighted versus myopic players) on the coordination problem. We propose the notion of myopic-farsighted stable strategy profiles and we show that it refines the concept of Nash equilibrium. In addition, our analysis reveals how crucial it might be to have a precise knowledge of the social and economic network for implementing efficiently policies for improving coordination among the interacting agents.

The structure of the paper is as follows: In Section 2 we describe the coordination game played on a fixed network. In Section 3 we define the concept of farsightedly stable strategy profiles and we prove its existence. We then characterize the set of stable strategy profiles in the presence of either only moderate players, or moderate players with stubborn players for a single project, or moderate players together with stubborn players of both types. In Section 4 we introduce myopic players together with farsighted ones, and possibly stubborn players. We propose the concept of myopic-farsighted stable strategy profiles and we analyze the impact of farsightedness and stubbornness. In Section 5 we look at the role of the social planner whose objective is to improve coordination within the whole network. In Section 6 we conclude. All proofs are relegated to the appendix.

\section{Coordination problems}

\section{$2.1 \quad$ Networks}

Let $N=\{1, \ldots, n\}$ be a finite set of players who are located on a fixed connected network $g$. A network $g$ is a list of which pairs of players are linked to each other and $i j \in g$ indicates that $i$ and $j$ are linked under $g$. A path in a network $g$ between $i$ and $j$ is a sequence of players $i_{1}, \ldots, i_{K}$ such that $i_{k} i_{k+1} \in g$ for each $k \in\{1, \ldots, K-1\}$ with $i_{1}=i$ and $i_{K}=j$. A network $g$ is connected if for all $i \in N$ and $j \in N \backslash\{i\}$, there exists a

\footnotetext{
${ }^{5}$ Another related strand of literature where we observe similar network effects are local public good models that do analyze the provision of public goods in networks (see e.g. Bramoullé and Kranton, 2007; Allouch, 2015).
} 
path in $g$ connecting $i$ and $j$. Let $g^{N}$ be the complete network. Let $N(g)=\{i \mid$ there is $j$ such that $i j \in g\}$ be the set of players who have at least one link in the network $g$. Let $N_{i}(g)=\{j \in N \mid i j \in g\}$ be the set of neighbors of player $i$ in $g$. The degree of player $i$ in network $g$, denoted $d_{i}(g)$, is the cardinality of $i$ 's set of neighbors, $d_{i}(g)=\# N_{i}(g) .{ }^{6}$ A player who has only one neighbor is called a loose end. A star network is a network such that there exists some player $i$ (the center) who is linked to every other player $j \neq i$ (the peripherals) and that contains no other links (i.e. $g$ is such that $N_{i}(g)=N \backslash\{i\}$ and $N_{j}(g)=\{i\}$ for all $\left.j \in N \backslash\{i\}\right)$. A line network is a connected network where each player $i \in N \backslash\{j, k\}$ has two links while players $j$ and $k$ are loose ends.

\subsection{A coordination game played on a fixed network}

The society consists of $n$ players located on a fixed network who have to choose between two projects $p$ or $q$. For sake of exposition, we limit the analysis to two projects. However, all our main results hold if we would consider more than two projects. A project $r \in\{p, q\}$ is said to be successful if at least $t^{r}$ players choose this project. We call $t^{r}$ the support threshold of project $r \in\{p, q\}, 0<t^{r} \leq n$ and $t^{r} \in \mathbb{N}$. Let $t=\left(t^{p}, t^{q}\right)$. Some players are moderate, while others are stubborn. Each moderate player chooses one project or action $a_{i} \in\{p, q\}$. Let $N_{m}=\{1, \ldots, m\}$ be the set of moderate players. A stubborn player for project $r \in\{p, q\}$ always chooses the project $r$ whatever the choice of other players and the thresholds. Let $N_{r}$ be the set of stubborn players for project $r, r \in\{p, q\}$. Thus, if $i \in N_{r}$ then $a_{i}=r$. Let $N_{p} \cup N_{q}=\{m+1, \ldots, n\}$. Let $a=\left(a_{1}, \ldots, a_{n}\right)$ be a strategy profile and $A$ be the set of all possible strategy profiles. ${ }^{7}$ The set of players $N=\{1, \ldots, n\}$ is simply $N_{m} \cup N_{p} \cup N_{q}{ }^{8}$

Given a strategy profile $a \in A$, let $n_{i}(a)=\#\left\{j \in N \mid a_{i}=a_{j}\right\}$ be the total number of players who choose the same project as player $i$ (stubborn players as well as player $i$ included), and let $\bar{n}_{i}(g, a)=\#\left\{j \in N \mid a_{i}=a_{j}\right.$ and $\left.i j \in g\right\}$ be the number of neighbors (stubborn players included) of player $i$ in the network $g$ who choose the same project as player $i$. Given some network $g$, some thresholds $\left(t^{p}, t^{q}\right)$ and some strategy profile $a \in A$, the utility function $U_{i}$ of a moderate player $i \in N_{m}$ is given by the following expression:

$$
U_{i}\left(g ; a_{i}, a_{-i}\right)= \begin{cases}-c_{i} & \text { if } n_{i}\left(a_{i}, a_{-i}\right)<t^{a_{i}} \\ b_{i}+\phi_{i}\left(\bar{n}_{i}(g, a)\right) & \text { if } n_{i}\left(a_{i}, a_{-i}\right) \geq t^{a_{i}}\end{cases}
$$

where $c_{i} \geq 0$ is player $i$ 's loss from investing into an unsuccessful project while $b_{i}+$ $\phi_{i}\left(\bar{n}_{i}(g, a)\right)$ is player $i$ 's benefit from a successful project. This benefit is composed of

\footnotetext{
${ }^{6}$ Throughout the paper we use the notation $\subseteq$ for weak inclusion and $\varsubsetneqq$ for strict inclusion. Finally, \# will refer to the notion of cardinality.

${ }^{7}$ Notice that strategy profiles where some stubborn player for one project would choose the other project are excluded from the set of all possible strategy profiles $A$.

${ }^{8}$ For a $n \times 1$ vector $a$ we denote by $a_{-i}$ the vector of size $n-1$ with the $i$-th entry removed and we use $a_{-i, j}$ to denote the vector in which the $i$-th and the $j$-th elements have been deleted.
} 


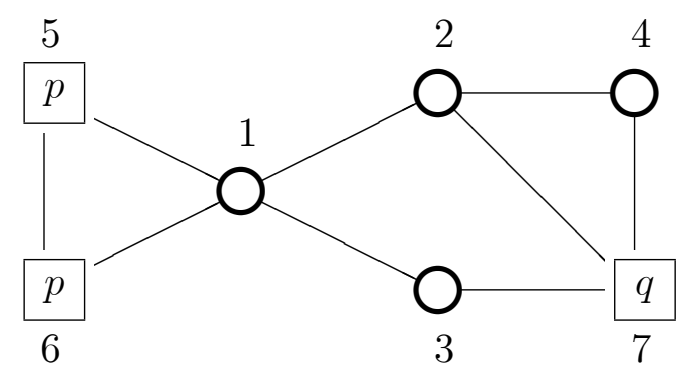

Figure 1: Network with four moderate players $(1,2,3,4)$ and three stubborn players $(5,6,7)$.

two parts: a fixed one $\left(b_{i}>0\right)$ and a variable one $\left(\phi_{i}\left(\bar{n}_{i}(g, a)\right) \geq 0\right)$ which depends on the action chosen by the neighbors of player $i$. The function $\phi_{i}$ is increasing with the number of neighbors who choose the same project as $i$ and it captures the fact that players may have different interests in cooperating with their neighbors. So, an investment of player $i$ into an unsuccessful project $\left(n_{i}\left(a_{i}, a_{-i}\right)<t^{a_{i}}\right)$ gives her a negative utility $\left(-c_{i}\right)$, while an investment into a successful project $\left(n_{i}\left(a_{i}, a_{-i}\right) \geq t^{a_{i}}\right)$ yields her a positive utility. Furthermore, in that case, the utility of player $i$ is increasing with the number of neighbors who choose the same project. ${ }^{9}$

Let us point out some properties of this utility function. In the case player $i$ chooses a successful project, her utility increases if some neighbor $j$ switches to her project: $U_{i}\left(g ; a_{i}, a_{j}, a_{-i, j}\right)>U_{i}\left(g ; a_{i}, a_{j}^{\prime}, a_{-i, j}\right)$ if $a_{j}^{\prime} \neq a_{j}=a_{i}, j \in N_{i}$ and $n_{i}\left(a_{i}, a_{j}, a_{-i, j}\right) \geq t^{a_{i}}$. The utility of player $i$ does not change if her neighbor, player $j$ changes her action as long as the project chosen by player $i$ stays unsuccessful: $U_{i}\left(g ; a_{i}, a_{-i}\right)=U_{i}\left(g ; a_{i}, a_{-i}^{\prime}\right)$ for any $a_{-i}$ and $a_{-i}^{\prime}$ if $n_{i}\left(a_{i}, a_{-i}\right)<t^{a_{i}}$ and $n_{i}\left(a_{i}, a_{-i}^{\prime}\right)<t^{a_{i}}$.

From now on we only consider projects that can be successful: $\# N_{m}+\# N_{r} \geq t^{r}$ for $r \in\{p, q\}$. It ensures that each project $r$ can be successful because there are not too many stubborn players for the other project such that the threshold of project $r$ cannot be reached even if all stubborn players for project $r$ and all moderate players invest into project $r$.

In Figure 1 we illustrate a situation with seven players located on a network who have to decide on investing into alternative projects $p$ or $q$. Players with a round node are moderate players, while a player with a square node is stubborn for the project written in the square. In this example there are two stubborn players for project $p$, namely players 5 and 6. Player 7 is stubborn for project $q$ while players 1, 2, 3 and 4 are moderate. Suppose players 1 and 2 choose $p$ while players 3 and 4 choose $q$. Then, $a=(p, p, q, q, p, p, q)$.

\footnotetext{
${ }^{9}$ For simplicity, we assume that a stubborn player for project $r \in\{p, q\}$ always chooses the project $r$ whatever the choice of other players and the thresholds. Such behavior would emerge endogenously by simply assuming that a stubborn player $i$ choosing the alternative project obtains a disutility of $-c_{i}-\bar{c}$ $(\bar{c}>0)$.
} 


\section{$3 \quad$ Farsighted agents}

\subsection{Farsightedly stable strategy profiles}

Typically, coordination games are solved using the concept of Nash equilibrium. A strategy profile $a^{*} \in A$ is a Nash Equilibrium if $U_{i}\left(g ; a_{i}^{*}, a_{-i}^{*}\right) \geq U_{i}\left(g ; a_{i}, a_{-i}^{*}\right)$ for all $i \in N_{m}$, for all $a_{i} \in\{p, q\}$. Let $\mathcal{N}(g, t)$ denote the set of all Nash Equilibria.

Assume now that players are farsighted. A farsighted player foresees that once she makes a choice and deviates, other players may deviate afterwards. A farsighted improving path is a sequence of strategy profiles that can emerge when farsighted players choose an action based on the improvement the end strategy profile offers them relative to the current strategy profile and where each strategy profile in the sequence differs from the preceding one in that only one action of one of the players is modified.

Definition 1. Consider $(g, t)$ as given. A farsighted improving path from a strategy profile $a \in A$ to a strategy profile $a^{\prime} \in A$ is a finite sequence of strategy profiles $a^{0}, \ldots, a^{L} \in A$ with $a^{0}=a, a^{L}=a^{\prime}$ and $a^{j} \neq a^{k}$ for all $j, k \in\{1, \ldots, L-1\}$ such that for every $\ell \in\{0, \ldots, L-1\}$, we have $a_{i}^{\ell+1} \neq a_{i}^{\ell}$ and $a_{-i}^{\ell+1}=a_{-i}^{\ell}$ for some $i \in N$, and $U_{i}\left(a^{L}\right)>U_{i}\left(a^{\ell}\right)$.

If there exists a farsighted improving path from the strategy profile $a$ to $a^{\prime}$ we write $a \rightarrow a^{\prime}$. The set of all strategy profiles that can be reached from $a$ by a farsighted improving path is denoted by $f(a)=\left\{a^{\prime} \in A \mid a \rightarrow a^{\prime}\right\}$. Our notion of farsighted improving path is similar to the one first developed by Jackson (2008) and Herings, Mauleon and Vannetelbosch (2009) for network formation.

Example 1. Consider the network depicted in Figure 2. All players are farsighted. Players 6 and 7 are stubborn while all other players are moderate. Both projects $p$ and $q$ have a low threshold: $t^{p}=t^{q}=1$. Start with the profile $a^{0}=(p, p, p, p, p, p, q)$ where player 5 is coordinating with one of her two neighbors. From this profile there is a farsighted improving path initiated by player 5 . First, player 5 chooses project $q$ instead of $p$ which yields to the profile $a^{1}=(p, p, p, p, q, p, q)$. In this profile $a^{1}$ player 3 has now an incentive to deviate to project $q$ as well, because two of her three neighbors have selected project $q$. This yields to the profile $a^{2}=(p, p, q, p, q, p, q)$. We observe that in $a^{2}$ player 5 is coordinating with both of her neighbors. Since she is farsighted, she had the incentive to start the improving path, taking into account that player 3 will follow her example and deviate from project $p$ to $q$ afterwards. This makes player 5 in profile $a^{2}$ better off than in $a^{0}$, even though she did not immediately (in $a^{1}$ ) benefit from her initial change.

Farsighted players anticipate the changes of the other players and compare their current utility with the utility they would get at the end of the improving path. Nash equilibrium requires strategy profiles to be immune to immediate deviations and does not capture that farsighted players anticipate the actions of other players and consider the end strategy profile that their deviations may lead to. To be stable, a strategy profile 


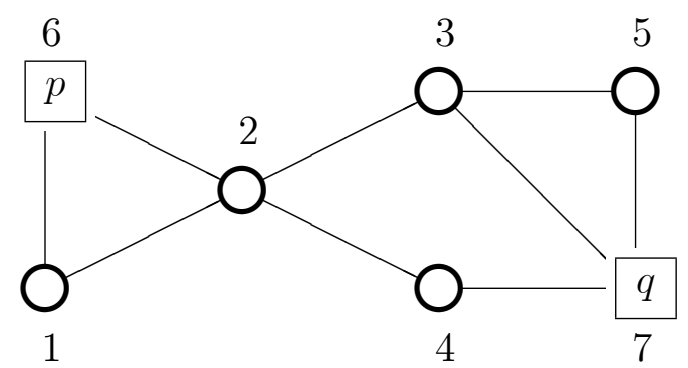

Figure 2: Network with five moderate players $(1,2,3,4,5)$ and two stubborn players $(6,7)$.

should be immune to deviations of farsighted players. These considerations lead to the following definition of farsightedly stable strategy profiles.

Definition 2. Consider $(g, t)$ as given. A strategy profile $a \in A$ is farsightedly stable if it satisfies $f(a)=\emptyset$.

A strategy profile $a \in A$ is farsightedly stable if there is no farsighted improving path leaving $a$. Let $Z(g, t)=\{a \in A \mid f(a)=\emptyset\}$ be the set of farsightedly stable strategy profiles. $^{10}$

Example 2. We reconsider the network depicted in Figure 2 where players 6 and 7 are stubborn while all other players are moderate. We still assume that $t^{p}=t^{q}=1$. Take $a^{*}=(p, p, q, q, q, p, q)$ where players 1 and 2 choose $p$ as the stubborn player 6 , while players 3, 4 and 5 choose project $q$ as the stubborn player 7 . We now argue that there are no farsighted improving path emanating from $a^{*}$. That is, $f\left(a^{*}\right)=\emptyset$. From any $a \in A$ such that $a_{5}=q$ player 5 will never change her action since there is no $a^{\prime} \in A$ where she would be strictly better off. Similarly, for player 1 when choosing project $p$ and for player 4 when choosing project $q$. Anticipating that player 5 will never switch her action, player 3 cannot be better off by choosing $p$ instead of $q$. Hence, player 3 will stick to project $q$. Finally, given that no other player will initiate a move from $(p, \cdot, q, q, q, p, q)$ and that player 2 is indifferent between $(p, p, q, q, q, p, q)$ and $(p, q, q, q, q, p, q)$, player 2 will not deviate from $(p, p, q, q, q, p, q)$. So, $f\left(a^{*}\right)=\emptyset$ and $(p, p, q, q, q, p, q)$ is a farsightedly stable strategy profile.

\subsection{Existence of a farsightedly stable strategy profile}

We now show that there always exists a farsightedly stable strategy profile, i.e., $Z$ is never empty, and we provide a method to find a farsightedly stable strategy profile. Starting from an arbitrary profile $a$, we can start writing the decision-making process of a player along a farsighted improving path as a tree. At each node one player decides whether she wants to stay with her current action or wants to deviate to another action. Since players can change their action many times along a farsighted improving path, the decision order

\footnotetext{
${ }^{10}$ Similarly, Jackson (2008) defines the concept of farsightedly pairwise stable networks. Farsightedly pairwise stable networks are the networks from which no farsighted improving path is emanating of it.
} 
of the players can be neglected. The order only decides which profile is reached, but if there is a farsighted improving path from $a$ when players decide in the order 1 to $m$, then there is also one for every other decision order. This is due to the fact that players can repeatedly change their actions. Still, it is not possible to illustrate an entire farsighted improving path as a tree, because there is an infinite number of nodes. Again, the reason is the possibility of repeated deviations from each player.

The following lemma allows us to write down the decision-making process as a finite tree when all players are farsighted.

Lemma 1. If there exists a farsighted improving path from profile a to profile $a^{\prime}$ then there also exists a farsighted improving path from a to $a^{\prime}$ in which each player changes her action at most once.

Lemma 1 implies that instead of considering all farsighted improving paths, we can focus our attention on farsighted improving paths in which each player changes her action once or not at all. To find a farsightedly stable strategy profile we formulate a game tree. First, we need to fix a decision order among the moderate players, without loss of generality $(1, \ldots, m)$ and we select an arbitrary profile $a^{0}$. Then, we can start to create the decision tree. In the first node player 1 decides if she wants to stay with her action $a_{1}^{0}$ or if she wants to deviate to any other action. In case of indifference we assume she stays. If she deviates, we reach a new profile $a^{1}$ with $a_{1}^{0} \neq a_{1}^{1}$ and $a_{-1}^{0}=a_{-1}^{1}$. In that case we remove player 1 from the decision order and we start from the beginning of the updated order, in this case with player 2. Player 2 then faces the same choice, staying in $a^{1}$ or deviating. If she deviates we move to $a^{2}$, remove player 2 from the decision order and start from the beginning of the order. If player $i$ does not deviate, but stays in profile $a^{x}$, we move to the next player in the decision order and letting her decide whether she wants to deviate from $a^{x}$. When player $i$ does not change her decision, she stays in the decision order and can revisit her choice once another player has deviated. We reach a stable strategy profile either when we reach the end of the decision order and no player wants to deviate or when there are no players left in the decision order. To illustrate this game tree, we introduce the following notation. Let $o$ be the decision order of the moderate players, without loss of generality $(1, \ldots, m)$ and $o \backslash i$ implies that player $i$ is removed from the decision order, i.e. $o \backslash i=(1, \ldots, i-1, i+1, \ldots m)$. We generate the game tree for $\Gamma\left(a^{0}, o\right)$, i.e. starting with profile $a^{0}$ and order $o$ as shown in Figure 3. $\Gamma\left(a^{k}, \emptyset\right)$ gives the utility $U_{i}\left(a^{k}\right)$ to player $i$ where $\Gamma\left(a^{k}, \emptyset\right)$ represents the profile reached once all moderate players have been removed from the decision order or they don't want to deviate.

By construction, the finite game tree of $\Gamma\left(a^{0}, o\right)$ shows all the possible improving paths emanating from $a^{0}$ in which each farsighted moderate player deviates at most once. Together with Lemma 1 this yields the following result.

Theorem 1. The subgame perfect Nash equilibrium of the game $\Gamma\left(a^{0}, o\right)$ as defined above is a farsightedly stable profile. 


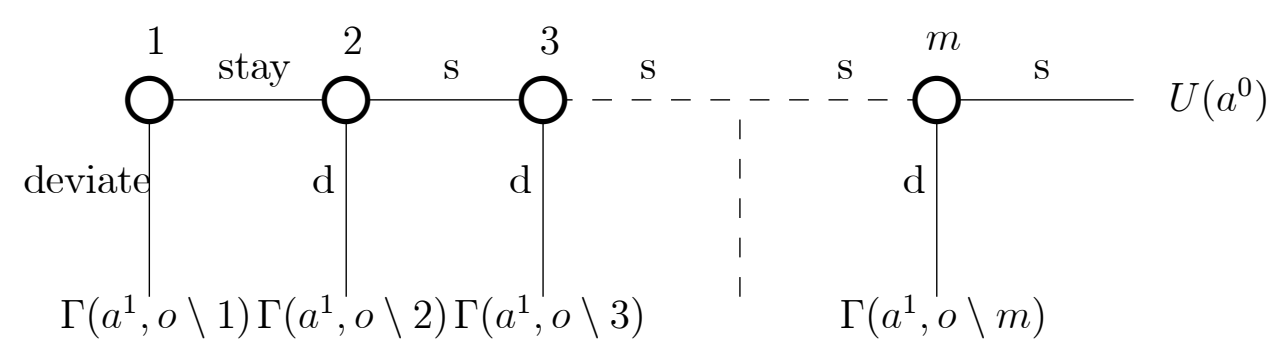

Figure 3: Game tree of $\Gamma\left(a^{0}, o\right)$.

Theorem 1 implies that there always exists at least one farsightedly stable strategy profile, i.e. the set of farsightedly stable profiles is non-empty. Starting from different profiles $a^{0}$ or using different orders $o$ can result in different farsightedly stable strategy profiles.

Example 3. We reconsider the network depicted in Figure 2 where players 6 and 7 are stubborn while all other players are moderate. We still assume that $t^{p}=t^{q}=1$. We can start from the profile $a^{o}=(p, p, p, p, p, p, q) \in A$ and the decision order $o=(1, \ldots, 5)$. Starting from profile $a^{0}$ the players 1 to 4 have no incentive to deviate, but player 5 does. She will select project $q$ even though this action does not provide her an immediate improvement, because she is anticipating that afterwards player 3 will deviate to project $q$ in the game $\Gamma((p, p, p, p, q, p, q),(1, \ldots, 4))$. After players 5 and 3 have switched to $q$ we reach the game $\Gamma((p, p, q, p, q, p, q),(1,2,4))$ where none of the players 1,2 or 4 have any incentive to change her action. So, the profile $(p, p, q, p, q, p, q)$ is farsightedly stable.

If we start from the profile $(q, q, q, q, q, p, q)$ no player will deviate and so it is farsightedly stable.

\subsection{No stubborn agents}

Suppose there are no stubborn players (i.e. $N=N_{m}$ ) and all moderate players are farsighted. Remember that $t$ is such that $\# N_{m}+\# N_{r} \geq t^{r}$ for $r \in\{p, q\}$. Hence, $\# N_{m} \geq t^{r}$ for $r \in\{p, q\}$. Farsighted moderate players will then choose the same project whatever the connected network.

Proposition 1. Suppose there are no stubborn players and all moderate players are farsighted. Then, $Z(g, t)=\left\{a \in A \mid a_{i}=a_{j}\right.$ for all $\left.i, j \in N\right\}$.

Consider the line network of Figure 4 with $t^{p}=t^{q}=1$. Suppose that the eight players choose the strategy profile $(p, p, q, q, p, p, q, q)$. This strategy profile is a Nash equilibrium of the coordination game played on the line. Indeed, no player has an incentive to choose another project given the choices made by her neighbors. But, it is an inefficient Nash equilibrium that is Pareto-dominated by both $(p, p, p, p, p, p, p, p)$ and $(q, q, q, q, q, q, q, q)$. 


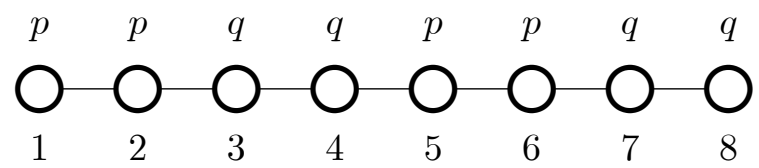

Figure 4: A line network with eight moderate players choosing a Pareto-dominated NE.

When players are farsighted they are able to coordinate on the same project and all inefficient Nash equilibria become unstable. Thus, without stubborn players, farsightedness solves the coordination problem.

\subsection{Only one type of stubborn agents}

Suppose there is only one type of stubborn players, without loss of generality for project $p$ (i.e. $N=N_{m} \cup N_{p}$ ), and all moderate players are farsighted. Remember that $t$ is such that $\# N_{m}+\# N_{p} \geq t^{p}$. All farsighted moderate players will then choose the project $p$ whatever the connected network.

Proposition 2. Suppose there are only stubborn players for project $p$ and all moderate players are farsighted, $N=N_{m} \cup N_{p}$. Then, $Z(g, t)=\left\{a \in A \mid a_{i}=p\right.$ for all $\left.i \in N\right\}=$ $\{(p, p, \ldots, p, p)\}$.

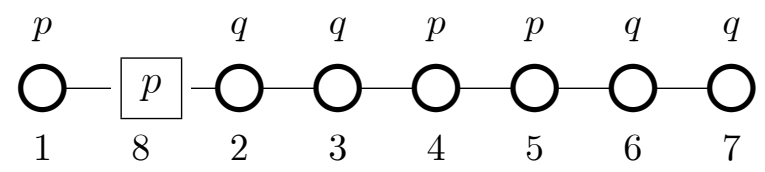

Figure 5: A line network with one stubborn for project $p$ and seven moderate players choosing a Pareto-dominated NE.

Consider the line network of Figure 5 with $t^{p}=t^{q}=1$. There is one stubborn player for project $p$ (player 8 ) and seven moderate players who choose the strategy profile $(p, q, q, p, p, q, q, p)$. This strategy profile is a Nash equilibrium of the coordination game played on the line. Indeed, no player has an incentive to choose another project given the choices made by her neighbors. But, it is an inefficient Nash equilibrium that is Paretodominated by the strategy profile $(p, p, p, p, p, p, p, p)$ where all moderate players choose the project $p$. Thus, in the presence of only one type of stubborn players, farsighted moderate player are able to coordinate on the project of the stubborn players and all inefficient Nash equilibria become unstable. Thus, with only one type of stubborn players, farsightedness still solves the coordination problem. 


\subsection{Both types of stubborn agents}

Suppose now that there are stubborn players for project $p\left(N_{p} \neq \emptyset\right)$ and stubborn players for project $q\left(N_{q} \neq \emptyset\right)$ as well as moderate players (i.e. $N=N_{m} \cup N_{p} \cup N_{q}$ ). Remember that all moderate players are farsighted and the threshold $t$ is such that $\# N_{m}+\# N_{r} \geq t^{r}$ for $r \in\{p, q\}$. We provide first a condition to guarantee that all moderate players fully coordinate on the same project, namely the one chosen by the larger group of stubborn players.

Proposition 3. If \# $\left(N_{i}(g) \cap N_{p}\right)>\#\left(N_{i}(g) \cap N_{q}\right)$ for all $i \in N_{m}$, then the strategy profile $a \in A$ with $a_{i}=p$ for all $i \in N_{m}$ is the unique farsightedly stable strategy profile.

We provide next a condition to guarantee that a subset of the moderate players coordinate on the same project.

Proposition 4. Suppose that $t^{r} \leq \# N_{r}+1$ for $r \in\{p, q\}$. If some moderate player $j \in N_{m}$ is such that $(\mathbf{i}) \#\left(N_{j}(g) \cap N_{p}\right)>\#\left(N_{j}(g) \cap N_{q}\right)$ and (ii) on all paths from $j$ to $i \in\left\{l \in N_{m} \mid \#\left(N_{l}(g) \cap N_{q}\right)>\#\left(N_{l}(g) \cap N_{p}\right)\right\}$ there is some stubborn player $k \in N_{p}$, then player $j$ chooses $a_{j}=p$ in any farsightedly stable strategy profile.

Proposition 4 tells us that a moderate player $j$ will choose the project $p$ if she is not influenced by stubborn players for project $q$ nor by moderate players who are themselves influenced by more stubborn players for project $q$ than for project $p$. In fact, all moderate players on the paths from $j$ up to reaching a first stubborn player for project $p$ (if any) will choose project $p$ since they also satisfy the condition of Proposition 4. Hence, all moderate players within the local network of player $j$ will coordinate on the project $p$.

Notice that if there is some $j \in N_{m}$ such that $\#\left(N_{j}(g) \cap N_{p}\right)>\#\left(N_{j}(g) \cap N_{q}\right)$ and on all paths from $j$ to $i \in\left\{l \in N_{m} \mid \#\left(N_{l}(g) \cap N_{q}\right)>\#\left(N_{l}(g) \cap N_{p}\right)\right\}$ there is some stubborn player $k \in N_{p}$, and there is some moderate player $j^{\prime} \in N_{m}$ such that $\#\left(N_{j^{\prime}}(g) \cap N_{q}\right)>$ $\#\left(N_{j^{\prime}}(g) \cap N_{p}\right)$ and on all paths from $j^{\prime}$ to $i \in\left\{l \in N_{m} \mid \#\left(N_{l}(g) \cap N_{p}\right)>\#\left(N_{l}(g) \cap N_{q}\right)\right\}$ there is some stubborn player $k \in N_{q}$, then both projects $p$ and $q$ will coexist and be adopted by moderate players in any farsightedly stable strategy profile.

\section{Myopic with farsighted agents}

Suppose now that we face a mixed population of moderate players in terms of their degree of farsightedness. That is, moderate players can be either farsighted or myopic. We denote by $M$ the set of myopic players and by $F$ the set of farsighted players. Obviously, we have $N_{m}=F \cup M$. Either set $(M$ or $F$ ) is allowed to be empty. A myopic-farsighted improving path is a sequence of strategy profiles that can emerge when farsighted players choose an action based on the improvement the end strategy profile offers them relative to the current strategy profile while myopic players choose an action based on the improvement the resulting strategy profile offers them relative to the current one. Each strategy profile 
in the sequence differs from the preceding one in that only one action of one of the players is modified.

Definition 3. Consider $(g, t)$ as given. A myopic-farsighted improving path from a strategy profile $a \in A$ to a strategy profile $a^{\prime} \in A$ is a finite sequence of strategy profiles $a^{0}, \ldots, a^{L} \in A$ with $a^{0}=a, a^{L}=a^{\prime}$ and $a^{j} \neq a^{k}$ for all $j, k \in\{1, \ldots, L-1\}$ such that for every $\ell \in\{0, \ldots, L-1\}$, we have $a_{i}^{\ell+1} \neq a_{i}^{\ell}$ and $a_{-i}^{\ell+1}=a_{-i}^{\ell}$ for some $i \in N$, and $U_{i}\left(a^{\ell+1}\right)>U_{i}\left(a^{\ell}\right)$ if $i \in M$ or $U_{i}\left(a^{L}\right)>U_{i}\left(a^{\ell}\right)$ if $i \in F$.

If there exists a myopic-farsighted improving path from the strategy profile $a$ to $a^{\prime}$ we write $a \longrightarrow a^{\prime}$. The set of all strategy profiles that can be reached from $a$ by a myopic-farsighted improving path is denoted by $h(a)=\left\{a^{\prime} \in A \mid a \longrightarrow a^{\prime}\right\}$. Our notion of myopic-farsighted improving path is similar to the one first developed by Herings, Mauleon and Vannetelbosch (2017) for two-sided matching problems. When all players are myopic, this notion of myopic-farsighted improving path is similar to the notion of improving path proposed by Jackson and Watts (2002b) for network formation.

Example 4. Consider again the network depicted in Figure 2. All players are myopic. Players 6 and 7 are stubborn while all other players are moderate. Player 6 is stubborn for project $p$ while player 7 is stubborn for project $q$. Both projects have low thresholds: $t^{p}=t^{q}=1$. Take the profile $a^{0}=(q, q, p, p, p, p, q)$ where players 3,4 and 5 choose project $p$ and players 1 and 2 select $q$. An example for an improving path from this profile is the following one. Player 2 has only one neighbor (player 1) with the same choice as her and three neighbors who choose project $p$. So it is an improvement for her to select $p$ as well. From this profile $a^{1}=(q, p, p, p, p, p, q)$ there is a second step towards $a^{2}=(p, p, p, p, p, p, q)$. Since player 2 is now choosing project $p$, player 1 is not coordinating with any neighbor. She is better off if she switches her choice to project $p$. All players are myopic and consider the immediate improvement they get from changing their action. From $a^{2}=(p, p, p, p, p, p, q)$ we have $h\left(a^{2}\right)=\emptyset$. This is different if we consider farsighted players.

Suppose now that player 5 becomes farsighted. Start with the profile $a^{0}=(p, p, p, p, p, p, q)$ where player 5 is coordinating with one of her two neighbors. From this profile there is an improving path initiated by player 5 . First, player 5 chooses project $q$ instead of $p$ which yields to the profile $a^{1}=(p, p, p, p, q, p, q)$. In this profile $a^{1}$ player 3 has now an incentive to deviate to project $q$ as well, because two of her three neighbors have selected project $q$. This yields to the profile $a^{2}=(p, p, q, p, q, p, q)$. We observe that in $a^{2}$ player 5 is coordinating with both of her neighbors. Since she is farsighted, she had the incentive to start the improving path, taking into account that player 3 will follow her example and deviate from project $p$ to $q$ afterwards. This makes player 5 in profile $a^{2}$ better off than in $a^{0}$, even though she did not immediately (in $a^{1}$ ) benefit from her initial change.

Only farsighted players anticipate the changes of the other players and compare their current utility with the utility they would get at the end of the improving path. Nash 
equilibrium requires strategy profiles to be immune to immediate deviations and does not capture that farsighted players anticipate the actions of other players and consider the end strategy profile that their deviations may lead to. In order to be stable, a strategy profile should be immune to deviations of both myopic and farsighted players. These considerations lead to the following definition of myopic-farsighted stable strategy profiles.

Definition 4. Consider $(g, t)$ as given. A strategy profile $a \in A$ is a myopic-farsighted stable profile if it satisfies $h(a)=\emptyset$.

A strategy profile $a \in A$ is a myopic-farsighted stable profile if there is no myopicfarsighted improving path leaving $a$. Let $\widehat{Z}(g, t)=\{a \in A \mid h(a)=\emptyset\}$ be the set of myopic-farsighted stable strategy profiles.

Let us first analyze some properties of the set of myopic-farsighted stable strategy profiles. The next proposition establishes that there are no cycles. That is, starting at $a$ there is no myopic-farsighted improving path that ends at $a$.

Proposition 5. Take any $a \in A$. We have $a \notin h(a)$.

Notice that if $a^{*} \in \mathcal{N}(g, t)$ is a Nash equilibrium, then there is no profitable individual deviation from $a^{*}$. Thus, $h\left(a^{*}\right)=\emptyset$ when all players are myopic, i.e. $N_{m}=M$. As a corollary of Proposition 5, we have that there always exist a myopic-improving path from any strategy profile $a \notin \mathcal{N}(g, t)$ to some Nash equilibrium strategy profile $a^{*} \in \mathcal{N}(g, t)$.

Corollary 1. Suppose that all players are myopic, $N_{m}=M$. Then, for any strategy profile $a \notin \mathcal{N}(g, t)$, there exists a Nash equilibrium strategy profile $a^{*} \in \mathcal{N}(g, t)$ such that $a^{*} \in h(a)$.

From Proposition 5 and Corollary 1 we obtain the next theorem.

Theorem 2. Suppose that all players are myopic, $N_{m}=M$. A set of strategy profiles is a set of myopic-farsighted stable strategy profiles if and only if it is equal to the set of Nash equilibrium strategy profiles.

Theorem 2 states an important result as it shows the connection between the set of myopic-farsighted stable strategy profiles and the set of Nash equilibria. As long as all moderate players are myopic the sets are the same. Thus, if $N_{m}=M$ then $\widehat{Z}(g, t)=$ $\mathcal{N}(g, t)$.

\subsection{Impact of farsightedness}

What happens to the set of myopic-farsighted stable strategy profiles when some myopic moderate players become farsighted or vice-versa? Let $\widehat{Z}(g, t, M, F)$ be the set of myopic-farsighted stable strategy profiles when $N_{m}=M \cup F$. Take some myopic (farsighted) player $i \in M(i \in F)$. We next show that the set of myopic-farsighted stable strategy profiles weakly decreases (increases) when a myopic (farsighted) player $i$ becomes farsighted (myopic). That is, $\widehat{Z}\left(g, t, M^{\prime}=M \backslash\{i\}, F^{\prime}=F \cup\{i\}\right) \subseteq \widehat{Z}(g, t, M, F)$ and $\widehat{Z}(g, t, M, F) \subseteq \widehat{Z}\left(g, t, M^{\prime}=M \cup\{i\}, F^{\prime}=F \backslash\{i\}\right)$. 
Theorem 3. $\widehat{Z}\left(g, t, M^{\prime}=M \backslash\{i\}, F^{\prime}=F \cup\{i\}\right) \subseteq \widehat{Z}(g, t, M, F)$.

Theorem 3 implies that the set of stable profiles weakly decreases when a myopic player turns farsighted. From Theorem 1 we know that even though the set weakly decreases it will never become empty since $\widehat{Z}\left(g, t, M=\emptyset, F=N_{m}\right)=Z(g, t) \neq \emptyset$. One immediate conclusion from Theorem 3 is that, if players $i$ and $j$ become farsighted, the order in which they become farsighted does not matter for the set of stable strategy profiles. Take $\widehat{Z}(g, t, M, F)$ with $i, j \in M$. We have:

$$
\begin{array}{ll}
\widehat{Z}(g, t, M \backslash\{i, j\}, F \cup\{i, j\}) & \subseteq \widehat{Z}(g, t, M \backslash\{i\}, F \cup\{i\}) \subseteq \widehat{Z}(g, t, M, F) ; \\
\widehat{Z}(g, t, M \backslash\{i, j\}, F \cup\{i, j\}) & \subseteq \widehat{Z}(g, t, M \backslash\{j\}, F \cup\{j\}) \subseteq \widehat{Z}(g, t, M, F) .
\end{array}
$$

From Theorem 3 we obtain the following corollary.

Corollary 2. The set of myopic-farsighted stable strategy profiles when there are both myopic and farsighted players $\widehat{Z}(g, t, M, F)$ is a subset of the set of Nash equilibrium strategy profiles $\mathcal{N}(g, t)$. That is, $\widehat{Z}(g, t, M, F) \subseteq \mathcal{N}(g, t)$.

Thus, in our coordination game played on a fixed network, the set of myopic-farsighted stable strategy profiles when moderate players are both myopic and farsighted is a refinement of the set of Nash equilibrium strategy profiles.

Example 5. Consider again the network depicted in Figure 2. Player 6 is stubborn for project $p$ while player 7 is stubborn for project $q$. Both projects have low thresholds: $t^{p}=t^{q}=1$. When all moderate players are myopic, there are six stable (or Nash equilibrium) strategy profiles:

$$
\begin{aligned}
& a^{1}=(p, p, p, p, p, p, q), a^{2}=(p, p, p, q, p, p, q), a^{3}=(p, p, q, p, q, p, q) \\
& a^{4}=(p, p, q, q, q, p, q), a^{5}=(p, q, q, q, q, p, q), a^{6}=(q, q, q, q, q, p, q) .
\end{aligned}
$$

Turning any of the players 2 to 4 into farsighted players, does not change the set of stable strategy profiles at all. But, if player 5 becomes farsighted, the first two profiles $a^{1}$ and $a^{2}$ are not stable any more. In those profiles player 5 deviates from project $p$ to project $q$ and takes into account that player 3 then will change to $q$ as well. Even when all players are farsighted the profiles $a^{3}, a^{4}, a^{5}$ and $a^{6}$ remain stable.

\subsection{Impact of stubbornness}

In the example of Figure 2, there was one stubborn player for project $p$, one stubborn player for project $q$ and the remaining players were moderate. We can clearly observe that the set of myopic-farsighted stable strategy profiles does not only depend on the farsightedness of the moderate players, but also on the type of stubborn players and their position in the network.

Let $\widehat{Z}\left(g, t, N_{m}, N_{p}, N_{q}\right)$ be the set of myopic-farsighted stable strategy profiles when $N_{m}$ is the set of moderate players, $N_{p}$ is the set of stubborn players for project $p$ and $N_{q}$ 
is the set of stubborn players for project $q$. Let $\widehat{Z}\left(g, t, N_{m} \backslash\{i\}, N_{p} \cup\{i\}, N_{q}\right)$ be the set of myopic-farsighted stable strategy profiles when a moderate player $i$ becomes stubborn for project $p$. Obviously, we have that $\widehat{Z}\left(g, t, N_{m}, N_{p}, N_{q}\right) \subseteq\left(\widehat{Z}\left(g, t, N_{m} \backslash\{i\}, N_{p} \cup\{i\}, N_{q}\right)\right) \cup$ $\left(\widehat{Z}\left(g, t, N_{m} \backslash\{i\}, N_{p}, N_{q} \cup\{i\}\right)\right)$. If choosing a project $p$ is part of a stable strategy profile $a$ when player $i$ is moderate, then the strategy profile $a$ is still stable once player $i$ becomes stubborn for project $p$, while keeping fixed the type (moderate or stubborn) of the other players. That is, if $a \in \widehat{Z}\left(g, t, N_{m}, N_{p}, N_{q}\right)$ with $i \in N_{m}$ and $a_{i}=p$ then $a \in \widehat{Z}\left(g, t, N_{m} \backslash\{i\}, N_{p} \cup\{i\}, N_{q}\right)$. In addition, if there exists no stable strategy profile in which player $i$, while being moderate, chooses project $p$, new profiles become stable when player $i$ is stubborn for project $p$.

Example 6. Consider again the network depicted in Figure 2. Assume that all players are myopic and thresholds are low, i.e. $t^{p}=t^{q}=1$. In the case that all players are moderate, there are eight different stable strategy profiles: $a^{1}=(p, p, q, p, q, p, q), a^{2}=$ $(p, p, q, q, q, p, q), a^{3}=(p, q, q, q, q, p, q), a^{4}=(p, p, p, p, p, p, p), a^{5}=(q, q, p, q, p, q, p)$, $a^{6}=(q, q, p, p, p, q, p), a^{7}=(q, p, p, p, p, q, p), a^{8}=(q, q, q, q, q, q, q)$. Turning player 7 into a stubborn player for project $q$ keeps only those stable profiles in which player 7 chooses project $q$, namely: $a^{1}, a^{2}, a^{3}$ and $a^{8}$. In addition there are new stable strategy profiles: $a^{9}=(p, p, p, p, p, p, q), a^{10}=(p, p, p, q, p, p, q)$ and $a^{11}=(q, p, p, p, p, q, q)$. We see that the profiles $a^{9}$ and $a^{11}$ are modifications from the profiles $a^{4}$ respectively $a^{7}$ : The actions of the players 1 to 6 in profile $a^{9}\left(a^{11}\right)$ are the same as in $a^{4}\left(a^{7}\right)$ only the action of player 7 is different. In the strategy profile $a^{10}$ there is a change from at least two players compared to the profiles $a^{1}$ to $a^{4}$ and $a^{5}$ to $a^{8}$. If, in addition, we make player 6 stubborn for project $p$ we get the same six stable strategy profiles as in Example 5: $a^{1}, a^{2}, a^{3}, a^{9}, a^{10}$ and $a^{12}=(q, q, q, q, q, p, q)$. Again, turning a player into a stubborn player makes some strategy profiles unstable, while it stabilizes others.

On the other hand turning a player into a stubborn player does not always create new stable strategy profiles. Looking again at Example 5 where all players are myopic and player $6(7)$ is stubborn for $p(q)$, there are six stable strategy profiles. If we turn player 2 into a stubborn player for project $p$ only those four profiles where she chooses project $p$ remain stable. The same happens when we turn her into a stubborn player for project $q$. There are only two stable strategy profiles, the ones in which player 2 invests into project $q$.

Proposition 6. Suppose that the thresholds for both projects are low, $t^{p}=t^{q}=1$. If on all paths on the network $g$ between two players $i$ and $j$ there is a stubborn player, then the choice of player $i$ and $j$ are independent of each other.

When thresholds are low we can simplify our analysis with the help of Proposition 6 . If there are two (or more) groups of players who are only connected to the other group(s) through paths that lead through at least one stubborn player we can split the graph into two (or more) components and analyze them separately. For instance, in the network $g$ 
depicted in Figure 6, players 1 and 4 are only connected through stubborn players (players 5 and 6), so instead of analyzing the stable profiles in $g$ we can separately analyze $g_{1}$ and $g_{6}$.

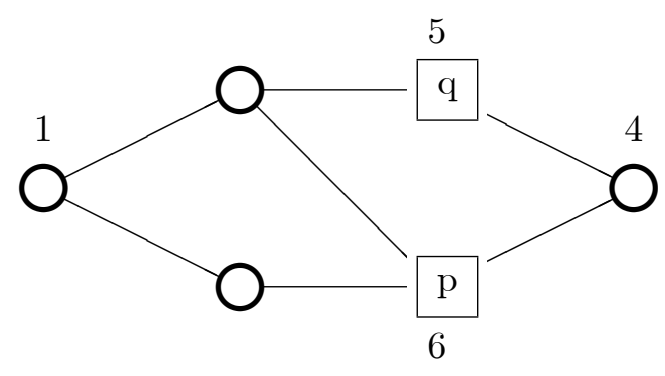

(a) $g$

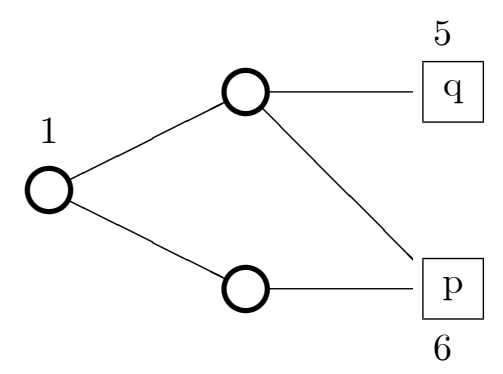

(b) $g_{1}$

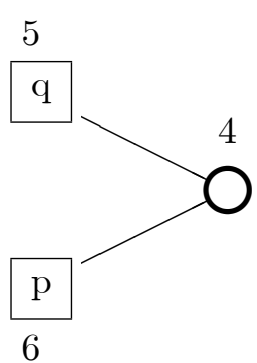

(c) $g_{4}$

Figure 6: An illustration of Proposition 6.

\subsection{Impact of the thresholds}

In addition to the types of the players, the thresholds for both projects have an impact on the stability of the strategy profiles. If we increase the threshold for project $p$ from $t^{p}$ to $t^{p}+1$, then some strategy profiles may not be stable, while other strategy profiles may become stable. For instance, suppose that the threshold for project $q$ is low, $t^{q}=1$. If there is $a \in \widehat{Z}\left(g,\left(t^{p}, 1\right), N_{m}, N_{p}, N_{q}\right)$ such that $\#\left\{i \in N_{m} \mid a_{i}=p\right\}+\# N_{p}=t^{p}$, then $a \notin \widehat{Z}\left(g,\left(t^{p}+1,1\right), N_{m}, N_{p}, N_{q}\right)$. If there is a strategy profile in which the threshold for $p$ is exactly reached, then an increase of the threshold for this project will make it unstable since moderate players have incentives to switch to the low threshold project $q$.

\subsection{Some specific networks}

The stability of a strategy profile does not only depend on the thresholds and the type of the players, but also on the network structure. We now analyze the set of stable strategy profiles for some common network configurations.

\subsubsection{Complete network}

In the case of the complete network, each player wants to coordinate with all other players. A moderate player wants to choose the project that gets chosen by the majority of the other players. This simplifies the analysis.

Proposition 7. Consider the complete network $g^{N}$ with low thresholds, $t^{p}=t^{q}=1$. We have $\widehat{Z}\left(g^{N}, t, N_{m}, N_{p}, N_{q}\right) \subseteq\left\{a \in A \mid a_{i}=a_{j}\right.$ for all $\left.i, j \in N_{m}\right\}$.

Proposition 7 confirms that the complete network leads to a full coordination of the moderate players. There might be at most two stable profiles depending on the number of stubborn players for each project and on the number of farsighted players. But, in all of 
them the moderate players choose the same action. For instance, if $\# N_{p}>\# N_{q}-1$ and $\# F \geq \# M$ then $a \in A$ such that $a_{i}=p$ for all $i \in N_{m}$ is the unique myopic-farsighted stable strategy profile.

\subsubsection{Star networks}

In a star network the center of the star plays an important role. Depending on the farsightedness and the type (moderate or stubborn) of the player in the center, there can be different stable strategy profiles. Let player $i^{*}$ be the center of the star and suppose that project $q$ is the project with the most stubborn players $\left(\# N_{q}>\# N_{p}\right)$. Remember that $\# N_{m}+\# N_{r} \geq t^{r}$ for $r \in\{p, q\}$.

Proposition 8. Take a star network with $i^{*}$ being the center. Suppose that $t^{r}<n / 2$ for $r \in\{p, q\}$.

(i) If $i^{*} \in M$, then $\widehat{Z}(g, t)=\left\{a \in A \mid a_{i}=a_{j}\right.$ for all $\left.i, j \in N_{m}\right\}$;

(ii) If $i^{*} \in F, \# F+\# N_{q} \geq t^{q}$ and $\# N_{q}>\# N_{p}$, then $\widehat{Z}(g, t)=\left\{a \in A \mid a_{i}=q\right.$ for all $\left.i \in N_{m}\right\}$;

(iii) If $i^{*} \in N_{q}$ and $\# F+\# N_{q} \geq t^{q}$, then $\widehat{Z}(g, t)=\left\{a \in A \mid a_{i}=q\right.$ for all $\left.i \in N_{m}\right\}$.

Take any given strategy profile $a$. Since $t^{r}<n / 2$ for $r \in\{p, q\}$, the project chosen by the majority of the players in $a$ is successful (without loss of generality project $q$ ). Hence, a myopic moderate player in the center has incentives to choose the project $q$. Once she has adopted this project, all other moderate players who were choosing $p$ have now incentives to switch to $q$. Thus, all moderate players will fully coordinate on the same project in any stable strategy profile when the center is a myopic moderate player.

If the center is a farsighted moderate player (i.e. $i^{*} \in F$ ), then the choice of this player depends on the thresholds of the projects. If the project with the most stubborn players (without loss of generality project $q$ ) has a low threshold $\left(\# F+\# N_{q} \geq t^{q}\right.$ ), she chooses this project and anticipates that all other farsighted moderate players will follow her choice anticipating that all myopic moderate players will do the same afterwards. Through this choice she can coordinate with the highest possible amount of neighbors and the only stable strategy profile is that all moderate players choose the project $q$ (i.e. full coordination among the moderate players).

If the center is a stubborn for project $q$ (without loss of generality) and her project has a low threshold $\left(\# F+\# N_{q} \geq t^{q}\right.$ ), then farsighted moderate players have incentives to choose $q$ anticipating that, once all of them have chosen project $q$, this project is for sure successful. Afterwards, all myopic moderate players have now incentives to adopt project $q$ too. Hence, the only stable strategy profile is that all moderate players choose project $q$ (i.e. full coordination among the moderate players). 


\subsubsection{Line networks}

In a line network we can sort the players into two categories: the loose-ends (i.e. the players who have only one neighbor) and the in-between players (i.e. the players with two neighbors). We already know that in any stable profile moderate loose-ends choose the same project as their neighbor when thresholds are low. On the other hand, the in-between players can coordinate with one or two of their neighbors. When all players are myopic there exist many different stable profiles. In the case of low thresholds $\left(t^{p}=t^{q}=1\right)$, the line can be fragmented into groups of size 2 or larger. Players in each fragment choose the same project. If we consider a fragment of two moderate and myopic players, just turning one of them farsighted will increase the coordination. The farsighted player will choose the same project as her other neighbor (from a different fragment) and the myopic player will follow. In a fragment of three players we need to turn at least two of them farsighted in order to make the players in the fragment coordinate with players from a neighboring fragment. Similarly, if the fragment consists of $x$ players, at least $x-1$ players have to become farsighted to achieve coordination with the neighboring fragments. The following proposition tells us when full coordination occurs on a line network.

Proposition 9. Consider a line network with only moderate players and with low thresholds, $t^{p}=t^{q}=1$. $\widehat{Z}\left(g,(1,1), N_{m}=N\right)=\left\{a \in A \mid a_{i}=a_{j}\right.$ for all $\left.i, j \in N\right\}$ if and only if $\# M \leq 1$.

Thus, full coordination will always occur on a line network if and only if almost all players are farsighted.

\section{Social planner}

We now look at the coordination problem from the point of view of a social planner. The objective of the social planner is to maximize the number of players who choose the same project. We consider two policy instruments. Either the social planner can socialize players by increasing the number of connections between the players (i.e. linking players who are not yet directly connected) or the social planner can turn some myopic players into farsighted ones (through some educative plans). In the case of the complete network with low thresholds there is no need for an intervention of the social planner. The moderate players will always end up coordinating on the project chosen by the larger group of stubborn players. In the case of a star network with a myopic player in the center, the social planner just needs to turn this myopic player into a farsighted player for reaching the desired outcome.

From now on we analyze the use of those two instruments in the case of the circle network $g^{c}$. Remember that $\left\{i \in N \mid a_{i}=r\right\}$ is the set of players who choose project $r \in\{p, q\}, \# F$ is the number of farsighted players, and $\# L(g)$ is the number of added links in $g$. Both projects are assumed to have low thresholds, $t^{p}=t^{q}=1$. For instance, 
the social planner can either add a link $i j$ between $i$ and $j$ (if $i j \notin g$ ) or turn a myopic player $i$ into a farsighted one. Then, $M$ and $F$ become $M^{\prime}=M \backslash\{i\}$ and $F^{\prime}=F \cup\{i\}$.

Without loss of generality, we consider the coordination on project $p$. A strategy profile $a \in \widehat{Z}(g,(1,1))$ if and only if $a$ is such that either $a_{i}=p$ and $\left\{j \in N_{i}\left(g^{c}\right) \mid a_{j}=p\right\} \neq \emptyset$ or $a_{i}=q$ and $\left\{j \in N_{i}\left(g^{c}\right) \mid a_{j}=q\right\} \neq \emptyset$. Suppose that the players along the circle are ordered: $\left\{i_{1}, i_{2}, \ldots, i_{n}\right\}$. A fragment of the circle is a subset of the ordered set of players, $\left\{i_{k}, i_{k+1}, \ldots, i_{k+l}\right\}$, such that each player in the fragment chooses the same project. Let $S_{r}^{l}\left(g^{c}, a\right)$ be a fragment $l$ of the circle network $g^{c}$ where players choose project $r$ in $a$. Notice that $\# S_{r}^{l}\left(g^{c}, a\right) \geq 2$. Let $S_{r}\left(g^{c}, a\right)=\cup_{l} S_{r}^{l}\left(g^{c}, a\right)=\left\{j \in N_{m} \mid a_{j}=r\right\}$.

Proposition 10. Consider a circle network $g^{c}$. Suppose that all players are moderate, $N=N_{m}$, and both projects have low thresholds, $t^{p}=t^{q}=1$. Given some $a \in \widehat{Z}\left(g^{c},(1,1)\right)$, in order to have full coordination on project $p$, the minimal requirements in terms of how many myopic players should become farsighted and/or how many links should be added are:

(i) $\# F=\#\left\{i \in N \mid a_{i}=q\right\}-1$, or

(ii) $\# L\left(g^{c}\right)=\#\left\{i \in N \mid a_{i}=q\right\}-1$, or

(iii) $\# F$ and $\# L=\#\left\{i \in N \mid a_{i}=q\right\}-1-\# F$, where $\# F<\#\left\{i \in N \mid a_{i}=q\right\}-1$.

Part (i) of Proposition 10 is in line with Proposition 9. The social planner has to turn mostly all moderate players who choose project $q$ into farsighted players in order to reach full coordination on project $p$. Part (ii) tells us that the social planner needs the same number of links (i.e. equal to the number of farsighted players needed for full coordination) in order to make the network denser and end up in full coordination. For instance, consider a fragment of two players, both of them are myopic and do not change their action since it will not increase their utility. However, as soon as one of the players has an additional connection to a player whose project is $p$, she is better off by switching her action, because now she has two neighbors choosing project $p$ versus one choosing $q$. If the fragment includes three players, adding two links between the two peripheral players (in this fragment) and two players choosing the opposite project makes them to adopt project $p$. Obviously, the central player (in this fragment) joins them afterwards. Part (iii) of Proposition 10 is about the joint implementation of both instruments: turning myopic players into farsighted ones and creating links between players with opposite projects. The two instruments act as substitutes since increasing the number of farsighted players reduces the needed number of links and vice versa. Obviously, those numbers are related to the total number of players who choose $q$.

To study the impact of stubbornness on the policies adopted by social planner, we now introduce one stubborn player for project $q$ into the circle network $g^{c}$. Remember that the presence of stubborn players for project $p$ does not affect the decision of the social planner as long as the objective of the social planner is to coordinate on project $p$. 
Proposition 11. Consider a circle network $g^{c}$. Suppose that all players are moderate, $N=N_{m}$, except one player who is stubborn for project q, i.e. $N=N_{m} \cup N_{q}$ with $N_{q}=\left\{i^{q}\right\}$. Both projects have low thresholds, $t^{p}=t^{q}=1$. Given some $a \in \widehat{Z}\left(g^{c},(1,1)\right)$, in order to have full coordination on project $p$ among moderate players, the minimal requirements in terms of how many links should be added and how many myopic players should become farsighted are:

(i) $\# L=\#\left\{i \in N \mid a_{i}=q\right\}-1$, or

(ii) $\# F$ and $\# L=\#\left\{i \in N \mid a_{i}=q\right\}-1-\# F$, where $0<\# F \leq \#\left\{i \in N \mid a_{i}=\right.$ $q\}-1-\#\left\{j \in N_{i^{q}}\left(g^{c}\right) \mid a_{j}=q\right\}$.

Proposition 11 tells us that, once there is one player who is stubborn for the opposite project, the social planner cannot reach full coordination among the moderate players by only turning myopic players into farsighted ones. ${ }^{11}$ Consider any fragment with three players choosing $q$. If one of the players is stubborn, then even though the other two players are farsighted, they do not change their actions. Indeed, they know that their neighbor, the stubborn player, always choose project $q$, and they would end up with the same utility if they switch to project $p$. On the contrary, if the social planner links each moderate player who is a neighbor of the stubborn player to some player choosing project $p$, then both players would now have incentives to switch to $p$ once their moderate neighbors have all chosen $p$. So, it suggests that adding links or promoting socialization can overcome some drawbacks of stubbornness in the society. Allowing players to communicate with a larger number of players reduces the likelihood of miscoordination, even in the presence of stubborn players. This argument is in line with Proposition 7 that confirms that the complete network leads to full coordination of the moderate players.

When there is no stubborn player for project $q$ (Proposition 10), the order for implementing both instruments (turning a myopic player into farsighted and/or adding a link) does not matter. However, in the presence of a stubborn player for project $q$, turning myopic players into farsighted ones without adding links first cannot lead to full coordination among the moderate players. Thus, a fraction of links has to be established first to provide the right incentives to the farsighted players for switching to project $p$.

Example 7. Consider the circle network $g^{c}$ depicted in Figure 7. The circle networks in (a) and (b) illustrate the stable strategy profile $a^{0}=(. . p, q, q, q, q, q, p, .$.$) . There are$ $\# S_{q}\left(g^{c}, a^{0}\right)=\#\{1,2,3,4,5\}$ players who choose project $q ; S_{q}\left(g^{c}, a^{0}\right)=\{1,2,3,4,5\}$ is the set of players who choose project $q$ and $N \backslash S_{q}\left(g^{c}, a^{0}\right)$ is the set of players who choose project $p$. To reach full coordination in case (a), the social planner can turn the myopic players $\{1,2,4,5\}$ into farsighted ones and the myopic player 3 will adopt their choice afterwards. So, $F=\{1,2,4,5\}, \# F=4$, and we reach the stable profile $a^{5}=(. . p, p, p, p, p, p, p, .$.

\footnotetext{
${ }^{11}$ However, if there are only stubborn players for the project the social planner wants to coordinate on, then Proposition 10 applies.
} 


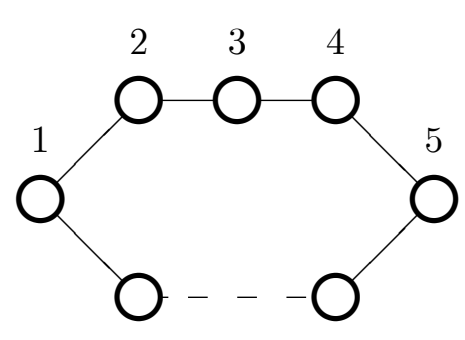

(a) $N=N_{m}$

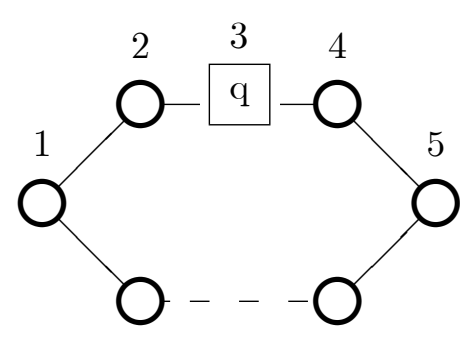

(b) $N=N_{m} \cup N_{q}$

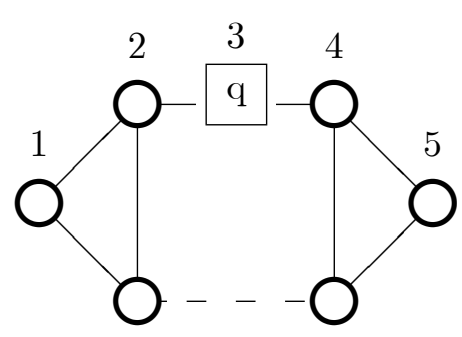

(c) $N=N_{m} \cup N_{q}$

Figure 7: An illustration of the impact of a stubborn player for project $q$.

with full coordination on project $p$ after five deviations from $a^{0}$ (see part (i) of Proposition 10). However, such a policy does not work when there is a stubborn player for project $q$. Consider the network (b), player 3 is now stubborn for project $q$. Suppose that the social planner turns players $\{1,2,4,5\}$ into farsighted players. Take $a^{2}=\{. . p, p, q, q, q, p, p, .$.$\} ,$ where players 1 and 5 have switched to $p$. But, at $a^{2}$, players 2 and 4 have no incentives to deviate since they anticipate that the stubborn neighbor 3 always chooses $q$. Therefore, $a^{0}$ is a stable strategy profile even with farsighted players. However, consider the network (c), where the social planner adds links to players 2 and 4 first, both have now three neighbors: two neighbors choosing project $q$ and one new neighbor choosing project $p$. Now turning players 1 and 5 into farsighted ones can lead to full coordination among the moderate players. Once players 1 and 5 are farsighted, they adopt project $p$, foreseeing that players 2 and 4 have then a majority of neighbors choosing $p$ and so they have incentives to switch to $p$ as well. Therefore, even though $\# F=2$ farsighted players initiate the deviation that leads to full coordination, it would not happen without first adding $\# L=2$ links to the circle network.

The analysis about the circle network stresses the complexity of looking at the optimal intervention of the social planner for avoiding miscoordination among the moderate players. Moreover, it illustrates that a very good knowledge of the network is necessary for implementing efficiently policy instruments to favor full coordination on some project.

\section{Conclusion}

We have studied a coordination game on a fixed connected network where players have to choose between two projects. Some players are moderate (i.e. they are ex-ante indifferent between both project) while others are stubborn (i.e. they always choose the same project). Benefits for moderate players are increasing in the number of neighbors who choose the same project. In addition, players are either farsighted or myopic. Farsighted players anticipate the reactions of others while myopic players do not. We have shown that, when all players are farsighted, full coordination among the moderate players is reached except if there are stubborn players for both projects. When all players are 
myopic the set of stable strategy profiles coincides with the set of Nash equilibria of the coordination game. When the population is mixed, the set of stable strategy profiles is a refinement of the set of Nash equilibrium strategy profiles. Turning myopic players into farsighted ones eliminates little by little the inefficient Nash equilibria. In fact, when some myopic players become farsighted, efficiency measured by the sum of the utilities weakly improves. We have restricted the analysis to two projects. However, all our main results (e.g. Theorem 1, Proposition 1, Proposition 2, Theorem 2, Theorem 3,...) are robust to more projects. Finally, we have considered a social planner who can improve coordination by means of two policy instruments: adding links to the network (socialization) and/or turning myopic players into farsighted ones (education). The objective of the social planner is to maximize the number of players who choose the same project. If there is at most one type of stubborn players, this objective does not conflict with the maximization of the sum of the utilities. However, once there are stubborn players for both projects, a tension may arise. Indeed, a group of players located in one part of the network may be better off by locally coordinating on some project, while another group of players located in another part of the network would be better off by locally coordinating on the other project. In this case, the only alternative for the social planner to reach full coordination would be to educate stubborn players to be moderate.

\section{Acknowledgements}

Ana Mauleon and Vincent Vannetelbosch are, respectively, Research Director and Senior Research Associate of the National Fund for Scientific Research (FNRS). Financial support from the MSCA ITN Expectations and Social Influence Dynamics in Economics (ExSIDE) Grant No721846 (1/9/2017-31/8/2020), from the Belgian French speaking community ARC project 15/20- 072 of Saint-Louis University - Brussels, and from the Fonds de la Recherche Scientifique - FNRS research grant T.0143.18 is gratefully acknowledged. We wish to thank Berno Buechel, Michel Grabisch, Agnieszka Rusinowska for useful comments as well as participants to the Stony Brook International Conference on Game Theory 2018, SING 2018, Fifth Annual Conference on Network Science and Economics 2019 (Indiana University, Bloomington), UECE Lisbon Meeting in Game Theory 2019.

\section{Appendix}

For the proofs of Lemma 1 and Proposition 5 we need the following lemmata about the preferences of the moderate players.

Lemma 2. Consider a strategy profile a with $a_{i} \neq a_{j}$ for some $i \neq j$. If at a, player $i$ prefers $a_{i}^{\prime}$ to $a_{i}$ (i.e. $\left.U_{i}\left(a_{i}^{\prime}, a_{-i}\right)>U_{i}\left(a_{i}, a_{-i}\right)\right)$ and at $\left(a_{i}^{\prime}, a_{-i}\right)$, player $j$ prefers $a_{j}^{\prime}$ to $a_{j}$ (i.e. $\left.U_{j}\left(a_{i}^{\prime}, a_{j}^{\prime}, a_{-i, j}\right)>U_{j}\left(a_{i}^{\prime}, a_{-i}\right)\right)$ with $a_{j}^{\prime} \neq a_{i}^{\prime}$, then it also holds that at a, player $j$ prefers $a_{j}^{\prime}$ to $a_{j}$ (i.e. $U_{j}\left(a_{j}^{\prime}, a_{-j}\right)>U_{j}\left(a_{j}, a_{-j}\right)$ ). 
Proof. We prove that $U_{j}\left(a_{j}^{\prime}, a_{-j}\right) \geq U_{j}\left(a_{i}^{\prime}, a_{j}^{\prime}, a_{-i, j}\right)>U_{j}\left(a_{i}^{\prime}, a_{-i}\right) \geq U_{j}\left(a_{j}, a_{-j}\right)$ holds. Since $a_{i}^{\prime} \neq a_{j}^{\prime}, a_{i} \neq a_{i}^{\prime}$ and $a_{j} \neq a_{j}^{\prime}$, we have $a_{i}=a_{j}^{\prime}$ and $a_{i}^{\prime}=a_{j}$. Hence, $U_{j}\left(a_{j}^{\prime}, a_{-j}\right) \geq$ $U_{j}\left(a_{i}^{\prime}, a_{j}^{\prime}, a_{-i, j}\right)$ and $U_{j}\left(a_{i}^{\prime}, a_{-i}\right) \geq U_{j}\left(a_{j}, a_{-j}\right)$ hold. If $i \in N_{j}(g)$ then the inequalities are strict.

The following lemma follows almost immediately and is presented without proof.

Lemma 3. Consider a strategy profile a from which player $i$ wants to deviate to $a_{i}^{\prime}$ and some other player $j, i \neq j$, wants to deviate to $a_{j}^{\prime}$, with $a_{j}^{\prime} \neq a_{i}^{\prime}$, i.e. $U_{i}\left(a_{i}^{\prime}, a_{-i}\right)>$ $U_{i}\left(a_{i}, a_{-i}\right)$ and $U_{j}\left(a_{j}^{\prime}, a_{-j}\right)>U_{j}\left(a_{j}, a_{-j}\right)$. Once the deviations have taken place, if player $i$ deviates again to $a_{i}$ (because $U_{i}\left(a_{i}, a_{j}^{\prime}, a_{-i, j}\right)>U_{i}\left(a_{i}^{\prime}, a_{j}^{\prime}, a_{-i, j}\right)$ ), then player $j$ will never switch back to $a_{j}$ (since $\left.U_{j}\left(a_{i}, a_{j}^{\prime}, a_{-i, j}\right)>U_{j}\left(a_{i}, a_{j}, a_{-i, j}\right)\right)$.

Proof of Lemma 1. It follows directly from Lemma 2 and Lemma 3.

Proof of Proposition 1. Since there are no stubborn players, $N=N_{m}$. (1) Take $a$ such that $a_{i}=a_{j}$ for all $i, j \in N$. Then, $f(a)=\emptyset$ since $U_{i}(a) \geq U_{i}\left(a^{\prime}\right)$ for all $i \in N$, for all $a^{\prime} \in A$. (2) Take any $a^{\prime} \neq a^{*}$ where $a^{*}$ is such that $a_{i}^{*}=p$ for all $i \in N$. We will show that $a^{*} \in f\left(a^{\prime}\right)$ and so $f\left(a^{\prime}\right) \neq \emptyset$. Let $a^{0}=a^{\prime}$ and $a^{L}=a^{*}$. We build a farsighted improving path from $a^{0}$ to $a^{L}$ as follows. For $l=1, \ldots, L-1$, let

$$
a^{l+1}= \begin{cases}a_{i}^{l+1}=a_{i}^{l}=p & \text { for all } i \in\left\{j \in N \mid a_{j}^{l}=p\right\} \\ a_{i^{l}}^{l+1}=p & \text { for } i=i^{l} \text { with } a_{i^{l}}^{l}=q \text { and } N_{i^{l}}(g) \cap\left\{j \in N \mid a_{j}^{l}=p\right\} \neq \emptyset \\ a_{i}^{l+1}=a_{i}^{l}=q & \text { for all } i \in\left\{j \in N \mid a_{j}^{l}=q\right\}, i \neq i^{l} .\end{cases}
$$

The sequence of strategy profiles $a^{0}, \ldots, a^{L}$ is a farsighted improving since along the sequence the player $i^{l}$ is such that $U_{i^{l}}\left(a^{L}\right)>U_{i^{l}}\left(a^{l}\right)$. Indeed, looking forward to $a^{*}$, player $i^{l}$ prefers $a^{*}$ to $a^{l}$ and so to switch to project $p$ anticipating that all players who haven't yet chosen $p$ will do it afterwards to finally reach the profile $a^{*}$. From (1) and (2) we have that $Z(g, t)=\left\{a \in A \mid a_{i}=a_{j}\right.$ for all $\left.i, j \in N\right\}$.

Proof of Proposition 2. Suppose there is only one type of stubborn players. Without loss of generality, let $N=N_{m} \cup N_{p}$ and $N_{q}=\emptyset$. We have that all stubborn players for project $p$ choose $p$, i.e. $a_{i}=p$ for all $i \in N_{p}$. The project $p$ can be successful since $\# N_{m}+\# N_{p} \geq t^{p}$. (1) Take $a^{*}$ such that $a_{i}^{*}=p$ for all $i \in N$. Then, $f\left(a^{*}\right)=\emptyset$ since $U_{i}\left(a^{*}\right) \geq U_{i}\left(a^{\prime}\right)$ for all $i \in N_{m}$, for all $a^{\prime} \in A$. (2) Take any $a^{\prime} \neq a^{*}$ where $a^{*}$ is such that $a_{i}^{*}=p$ for all $i \in N$. We will show that $a^{*} \in f\left(a^{\prime}\right)$ and so $f\left(a^{\prime}\right) \neq \emptyset$. Let $a^{0}=a^{\prime}$ and 
$a^{L}=a^{*}$. We build a farsighted improving path from $a^{0}$ to $a^{L}$ as follows. Notice that in $a^{0}$ we have $a_{i}^{0}=p$ for all $i \in N_{p}$. For $l=1, \ldots, L-1$, let

$$
a^{l+1}= \begin{cases}a_{i}^{l+1}=a_{i}^{l}=p & \text { for all } i \in\left\{j \in N \mid a_{j}^{l}=p\right\} \\ a_{i^{l}}^{l+1}=p & \text { for } i=i^{l} \text { with } a_{i^{l}}^{l}=q \text { and } N_{i^{l}}(g) \cap\left\{j \in N \mid a_{j}^{l}=p\right\} \neq \emptyset \\ a_{i}^{l+1}=a_{i}^{l}=q & \text { for all } i \in\left\{j \in N \mid a_{j}^{l}=q\right\}, i \neq i^{l} .\end{cases}
$$

The sequence of strategy profiles $a^{0}, \ldots, a^{L}$ is a farsighted improving since along the sequence the moderate player $i^{l}$ is such that $U_{i^{l}}\left(a^{L}\right)>U_{i^{l}}\left(a^{l}\right)$. Indeed, looking forward to $a^{*}$, player $i^{l}$ prefers $a^{*}$ to $a^{l}$ and so to switch to project $p$ anticipating that all players who haven't yet chosen $p$ will do it afterwards to finally reach the profile $a^{*}$. From (1) and (2) we have that $Z(g, t)=\left\{a \in A \mid a_{i}=p\right.$ for all $\left.i \in N\right\}=\{(p, p, p, \ldots, p, p, p)\}$.

Proof of Proposition 3. Suppose that there are stubborn players for project $p\left(N_{p} \neq \emptyset\right)$ and stubborn players for project $q\left(N_{q} \neq \emptyset\right)$ as well as moderate players (i.e. $N=$ $\left.N_{m} \cup N_{p} \cup N_{q}\right)$. The threshold $t$ is such that $\# N_{m}+\# N_{r} \geq t^{r}$ for $r \in\{p, q\}$. Suppose now that $\#\left(N_{i}(g) \cap N_{p}\right)>\#\left(N_{i}(g) \cap N_{q}\right)$ for all $i \in N_{m}$. We will show that the strategy profile $a^{*} \in A$ with $a_{i}^{*}=p$ for all $i \in N_{m}$ is the unique farsightedly stable strategy profile. (1) Take $a^{*}$ such that $a_{i}^{*}=p$ for all $i \in N_{m}$. Take any $i \in N_{m}$. Since $\#\left(N_{i}(g) \cap N_{p}\right)>$ $\#\left(N_{i}(g) \cap N_{q}\right)$ we have that $U_{i}\left(a^{*}\right) \geq U_{i}\left(a^{\prime}\right)$ for all $a^{\prime} \in A$. Hence, $f\left(a^{*}\right)=\emptyset$. (2) Take any $a^{\prime} \neq a^{*}$ where $a^{*}$ is such that $a_{i}^{*}=p$ for all $i \in N_{m}$. We will show that $a^{*} \in f\left(a^{\prime}\right)$ and so $f\left(a^{\prime}\right) \neq \emptyset$. Let $a^{0}=a^{\prime}$ and $a^{L}=a^{*}$. We build a farsighted improving path from $a^{0}$ to $a^{L}$ as follows. Notice that in $a^{0}$ we have $a_{i}^{0}=p$ for all $i \in N_{p}$ and $a_{i}^{0}=q$ for all $i \in N_{q}$. For $l=1, \ldots, L-1$, let

$$
a^{l+1}= \begin{cases}a_{i}^{l+1}=a_{i}^{l}=p & \text { for all } i \in\left\{j \in N_{m} \mid a_{j}^{l}=p\right\} \\ a_{i^{l}}^{l+1}=p & \text { for } i=i^{l} \text { with } a_{i^{l}}^{l}=q \text { and } N_{i^{l}}(g) \cap\left\{j \in N \mid a_{j}^{l}=p\right\} \neq \emptyset \\ a_{i}^{l+1}=a_{i}^{l}=q & \text { for all } i \in\left\{j \in N_{m} \mid a_{j}^{l}=q\right\}, i \neq i^{l} \\ a_{i}^{l+1}=a_{i}^{l}=q & \text { for all } i \in N_{q} \\ a_{i}^{l+1}=a_{i}^{l}=p & \text { for all } i \in N_{p}\end{cases}
$$

The sequence of strategy profiles $a^{0}, \ldots, a^{L}$ is a farsighted improving since along the sequence the moderate player $i^{l}$ is such that $U_{i^{l}}\left(a^{L}\right)>U_{i^{l}}\left(a^{l}\right)$ because of \# $\left(N_{i}(g) \cap N_{p}\right)>$ $\#\left(N_{i}(g) \cap N_{q}\right)$. Indeed, looking forward to $a^{*}$, player $i^{l}$ prefers $a^{*}$ to $a^{l}$ and to switch to project $p$ because she has more stubborn players for project $p$ than for project $q$ in her neighborhood and she anticipates that all moderate players who haven't yet chosen $p$ will do it afterwards to finally reach the profile $a^{*}$. From (1) and (2) we have that $Z(g, t)=\left\{a \in A \mid a_{i}=p\right.$ for all $i \in N_{m} \cup N_{p}, a_{i}=q$ for all $\left.i \in N_{q}\right\}$. 
Proof of Proposition 4. Suppose that $t^{r} \leq \# N_{r}+1$ for $r \in\{p, q\}$. Take some moderate player $j \in N_{m}$ such that (i) $\#\left(N_{j}(g) \cap N_{p}\right)>\#\left(N_{j}(g) \cap N_{q}\right)$ and (ii) on all paths from $j$ to $i \in\left\{l \in N_{m} \mid \#\left(N_{l}(g) \cap N_{q}\right)>\#\left(N_{l}(g) \cap N_{p}\right)\right\}$ there is some stubborn player $k \in N_{p}$. We will show that player $j$ chooses $a_{j}=p$ in any farsightedly stable strategy profile in $Z(g, t)$. That is, for any $a^{\prime}$ such that $a_{j}^{\prime}=q, f\left(a^{\prime}\right) \neq \emptyset$. Since on all paths from $j$ to $i \in\left\{l \in N_{m} \mid \#\left(N_{l}(g) \cap N_{q}\right)>\#\left(N_{l}(g) \cap N_{p}\right)\right\}$ there is some stubborn player $k \in N_{p}$, we have that on all paths from $j$ all players $l^{*}$ between $j$ and the first stubborn player on the path (if any) are moderate players who have more stubborn players for project $p$ than for project $q$ in their neighborhood. It follows that all those players $l^{*}$ and player $j$ (weakly) prefer the strategy profile $a^{\prime \prime}$ such that $a_{l}^{\prime \prime}=p, a_{j}^{\prime \prime}=p$ and $a_{i}^{\prime \prime}=a_{i}^{\prime}$ for all $i \in N_{m}, i \neq j, l^{*}$, to the strategy profile $a^{\prime}$. From $a^{\prime}=a^{0}$ we build a farsighted improving to $a^{\prime \prime}=a^{L}$ as follows. Take some player $l^{*}$ (or player $j$ ) such that $a_{l^{*}}^{\prime}=q$ and $N_{l^{*}}(g) \cap\left\{i \in N \mid a_{i}^{\prime}=p\right\} \neq \emptyset$. This moderate player $l^{*}$ is such that $U_{l^{*}}\left(a^{L}=a^{\prime \prime}\right)>U_{l^{*}}\left(a^{0}=a^{\prime}\right)$ and so she switches to project $p$ anticipating that the players in her neighborhood will do the same afterwards. Next some other player $l^{*}$ (or player $j$ ) such that $a_{l^{*}}^{\prime}=a_{l^{*}}^{0}=a_{l^{*}}^{1}=q$ and $N_{l^{*}}(g) \cap\left\{i \in N \mid a_{i}^{1}=p\right\} \neq \emptyset$. This moderate player $l^{*}$ is such that $U_{l^{*}}\left(a^{L}=a^{\prime \prime}\right)>U_{l^{*}}\left(a^{1}\right)$ and so she switches to project $p$ anticipating that the players in her neighborhood will do the same afterwards; and so forth until we reach the strategy profile $a^{L}=a^{\prime \prime}$ where $a_{l^{*}}^{\prime \prime}=p$ and $a_{j}^{\prime \prime}=p$. Hence, any $a \in Z(g, t)$ is such that player $j$ chooses $a_{j}=p$.

Proof of Proposition 5. We show that $a \notin h(a)$.

Suppose there exists a myopic-farsighted improving path $a^{0}, a^{1}, \ldots, a^{L}$ with $a=a^{0}$ and $a=a^{L}\left(a^{k} \neq a^{l}\right.$ for all $\left.k, l \in\{1, \ldots, L-1\}\right)$. Notice first that no farsighted player will initiate such a path. Only myopic players could move away from $a$. Let $i^{1}$ be the myopic player that deviates from $a^{0}$ to $a^{1}$, with $U_{i^{1}}\left(a_{i^{1}}^{1}, a_{-i^{1}}^{0}\right)>U_{i^{1}}\left(a_{i^{1}}^{0}, a_{-i^{1}}^{0}\right)$. Consider now the move of player $i^{2}$ from $a^{1}$ to $a^{2}$. If $a_{i^{2}}^{2}=a_{i^{1}}^{1}$, then player $i^{1}$ will not deviate back to $a_{i^{1}}^{0}$ since the support for the project she prefers is now even larger. Thus, as long as, along the path, the successive deviating players continue supporting project $a_{i^{1}}^{1}$, player $i^{1}$ will not deviate back to $a_{i^{1}}^{0}$ and then the end strategy profile of the improving path will never be equal to $a$.

Let $l$ be the first time along the path such that some myopic player $j$ deviates from $a^{l}$ to $a^{l+1}$, with $a_{j}^{l+1} \neq a_{i^{1}}^{1}=\ldots=a_{i^{l}}^{l}$ and $U_{j}\left(a_{j}^{l+1}, a_{i^{1}}^{1}, \ldots, a_{i^{l}}^{l}, a_{-j, i^{1}, \ldots, l^{l}}^{0}\right)>U_{j}\left(a_{i^{1}}^{1}, \ldots, a_{i^{l}}^{l}, a_{-i^{1}, \ldots, l^{l}}^{0}\right)$. Then by Lemma 2, we also have that player $j$ prefers $a_{j}^{l+1}$ to $a_{j}^{0}=a_{j}^{1}=\ldots=a_{j}^{l}$ before players $i^{1}, \ldots, i^{l}$ deviates from $a_{i^{1}}^{0}, \ldots, a_{i^{l}}^{l-1}$ to $a_{i^{1}}^{1}=\ldots=a_{i^{l}}^{l} \neq a_{j}^{l+1}$. It holds that

$$
\begin{aligned}
U_{j}\left(a_{j}^{l+1}, a_{i^{1}}^{1}, \ldots, a_{i^{l-1}}^{l-1}, a_{i^{l}}^{l}, a_{-j, i^{1}, \ldots, i^{l}}^{0}\right)> & U_{j}\left(a_{i^{1}}^{1}, \ldots, a_{i^{l}}^{l}, a_{-i^{1}, \ldots, i^{l}}^{0}\right), \\
U_{j}\left(a_{j}^{l+1}, a_{i^{1}}^{1}, \ldots, a_{i^{l-1}}^{l-1}, a_{-j, i^{1}, \ldots, l^{l-1}}^{0}\right)> & U_{j}\left(a_{i^{1}}^{1}, \ldots, a_{i^{l-1}}^{l-1}, a_{-i^{1}, \ldots, i^{l-1}}^{0}\right), \\
& \ldots \\
U_{j}\left(a_{j}^{l+1}, a_{-j}^{0}\right)> & U_{j}\left(a_{j}^{0}, a_{-j}^{0}\right) .
\end{aligned}
$$


Notice that, in order for the path to end at $a$, players $i^{1}, \ldots, i^{l-1}, i^{l}$ should have incentives to deviate back from $a_{i^{1}}^{1}=\ldots=a_{i^{l}}^{l}$ to $a_{i^{1}}^{0}, \ldots, a_{i^{l}}^{l-1}$, respectively. Thus, assume that this is the case once player $j$ (and possibly other players that deviate later on to the same project as player $j$ ) deviates from $a^{l}$ to $a^{l+1}$, with $a_{j}^{l+1} \neq a_{i^{1}}^{1}=\ldots=a_{i^{l}}^{l}$. But then, by Lemma 3, we have that player $j$ (and possibly some other player) will never deviate back to $a_{j}^{0} \neq a_{j}^{l+1}$. Hence, $a$ will never be reached.

Suppose now that, at $l$, a farsighted player $j$ deviates from $a^{l}$ to $a^{l+1}$, with $a_{j}^{l+1} \neq$ $a_{i^{1}}^{1}=\ldots=a_{i^{l}}^{l}$ and $U_{j}\left(a_{j}^{L}, a_{-j}^{L}\right)>U_{j}\left(a_{i^{1}}^{1}, \ldots, a_{i^{l}}^{l}, a_{-i^{1}, \ldots, i^{l}}^{0}\right)$. Then, in order to induce player $i^{1}$ to deviate back to $a_{i^{1}}^{0}$, the following case has to be considered: $a_{j}^{0}=a_{i^{1}}^{1}=\ldots=a_{i^{l}}^{l}$. We then obtain

$U_{j}\left(a_{j}^{0}, a_{-j}^{0}\right) \leq U_{j}\left(a_{i^{1}}^{1}, a_{j}^{0}, a_{-j, i^{1}}^{0}\right) \leq \ldots \leq U_{j}\left(a_{i^{1}}^{1}, \ldots, a_{i^{l}}^{l}, a_{j}^{0}, a_{-j, i^{1}, \ldots, i^{l}}^{0}\right)<U_{j}\left(a_{j}^{L}, a_{-j}^{L}\right)=U_{j}\left(a_{j}^{0}, a_{-j}^{0}\right)$,

a contradiction. Thus, no farsighted player along the path from $a$ to $a^{L}=a$ would be the first player deviating from $a^{l}$ to $a^{l+1}$, with $a_{j}^{l+1} \neq a_{i^{1}}^{1}=\ldots=a_{i^{l}}^{l}$.

Proof of Theorem 2. $(\Rightarrow)$ We show that the set of Nash equilibrium strategy profiles $\mathcal{N}(g, t)$ is a set of myopic-farsighted stable strategy profiles $\widehat{Z}(g, t)$. Let some $a^{*} \in \mathcal{N}(g, t)$ be given. It holds by definition of the Nash equilibrium that for all $a^{*} \in \mathcal{N}(g, t)$ we have $h\left(a^{*}\right)=\emptyset$. Hence, all $a^{*} \in \mathcal{N}(g, t)$ are myopic-farsighted stable strategy profiles. $(\Leftarrow) \mathrm{We}$ show that $\mathcal{N}(g, t)$ is the only set of myopic-farsighted stable strategy profiles $\widehat{Z}(g, t)$. Let some $a \notin \mathcal{N}(g, t)$ be given. By Corollary 1, we have that there exists a Nash equilibrium strategy profile $a^{*} \in \mathcal{N}(g, t)$ such that $a^{*} \in h(a)$. Hence, a strategy profile that is not a Nash equilibrium cannot be a myopic-farsighted stable strategy profile. Thus, if $N_{m}=M$ then $\widehat{Z}(g, t)=\mathcal{N}(g, t)$.

Proof of Theorem 3. Proof by contradiction. Suppose there is a strategy profile $a^{\prime} \in \widehat{Z}\left(g, t, M^{\prime}=M \backslash\{i\}, F^{\prime}=F \cup\{i\}\right)$ and $a^{\prime} \notin \widehat{Z}(g, t, M, F)$. This means that there is another profile $a=\left(a_{i}, a_{-i}\right)$ with $a_{-i}=a_{-i}^{\prime}$ such that $U_{i}(a)>U_{i}\left(a^{\prime}\right)$. So that the deviation from $a_{i}^{\prime}$ to $a_{i}$ is an immediate improvement for player $i$. Then, there is a myopic-farsighted improving path of length one from $a^{\prime}$ to $a$ when player $i$ is myopic, with $a \in h\left(a^{\prime}\right)$. But then, the previous myopic-farsighted improving path of length one from $a^{\prime}$ to $a$ is also a myopic-farsighted improving path of length one from $a^{\prime}$ to $a$ when player $i$ is farsighted. This implies that $a^{\prime}$ is not stable if $i$ is farsighted and contradicts our assumption. 
Proof of Proposition 6. Let $i^{r}$ be a stubborn player for project $r$. Without loss of generality, suppose that $i, j \in N_{i^{r}}(g)$ and there exists no other path between $i$ and $j$ (otherwise repeat the same arguments). Clearly $a_{i^{r}}$ is independent of $a_{i}$ and $a_{j}$, because $i^{r}$ always chooses $a_{i^{r}}=r$. This implies that $a_{i}$ and $a_{j}$ are independent of each other, that $a_{k}$ for $k \in N_{i}(g) \cap N_{m}$ is independent of $a_{j}$, that $a_{l}$ for $l \in N_{j}(g) \cap N_{m}$ is independent of $a_{i}$ and so on.

Proof of Proposition 7. Consider the complete network $g^{N}$ with $t^{p}=t^{q}=1$. We will show that $\widehat{Z}\left(g^{N}, t, N_{m}, N_{p}, N_{q}\right) \subseteq\left\{a \in A \mid a_{i}=a_{j}\right.$ for all $\left.i, j \in N_{m}\right\}$. We prove by contradiction that there cannot be some moderate players who choose project $p$ and other moderate players who choose project $q$. Take any $a \in A$ where some moderate players $i_{1}, \ldots, i_{m_{1}}$ choose project $p$ and other moderate players $j_{1}, \ldots, j_{m_{2}}$ choose project $q$ with $m=m_{1}+m_{2}$. That is, $\left\{j_{1}, \ldots, j_{m_{1}}\right\}=\left\{i \in N_{m} \mid a_{i}=p\right\}$ and $\left\{j_{1}, \ldots, j_{m_{2}}\right\}=\left\{i \in N_{m} \mid\right.$ $\left.a_{i}=q\right\}$. Suppose that $\left.\# N_{p}+\#\left\{i \in N_{m} \mid a_{i}=p\right\}\right\} \geq \# N_{q}+\#\left\{i \in N_{m} \mid a_{i}=q\right\}$. In the complete network, for all $i \in\left\{j_{1}, \ldots, j_{m 2}\right\}$ we have $\#\left(N_{i}\left(g^{N}\right) \cap\left(N_{p} \cup\left\{i \in N_{m} \mid a_{i}=\right.\right.\right.$ $p\}))>\#\left(N_{i}\left(g^{N}\right) \cap\left(N_{q} \cup\left\{i \in N_{m} \mid a_{i}=q\right\}\right)\right)$ since $N_{i}\left(g^{N}\right)=N \backslash\{i\}$. This implies that $U_{i}\left(a_{i}=p, a_{-i}\right)>U_{i}\left(a_{i}=q, a_{-i}\right)$. Since \# $\left(N_{p} \cup\left\{i \in N_{m} \mid a_{i}=p\right\}\right)>\#\left(N_{q} \cup\{i \in\right.$ $\left.\left.N_{m} \mid a_{i}=q\right\}\right)-1$, it follows that a myopic or farsighted player will deviate to project $p: \quad U_{i}\left(a_{i}^{\prime}=p, a_{-i}^{\prime}=a_{-i}\right)>U_{i}\left(a_{i}=q, a_{-i}\right)$ for all $i \in\left\{j_{1}, \ldots, j_{m 2}\right\}$. There is a myopicfarsighted improving from $a$ to $a^{\prime}$. Hence, $a^{\prime} \in h(a)$ and $h(a) \neq \emptyset$. Thus, $\left\{a \in A \mid a_{i}=a_{j}\right.$ for all $\left.i, j \in N_{m}\right\}$ are the only candidates for being myopic-farsighted stable strategy profiles.

Proof of Proposition 8. Take a star network with $i^{*}$ being the center. Suppose that $t^{r}<n / 2$ for $r \in\{p, q\}$.

(i) Suppose $i^{*} \in M$. (ia) Take any $a \notin\left\{a \in A \mid a_{i}=a_{j}\right.$ for all $\left.i, j \in N_{m}\right\}$ and suppose (without loss of generality) that $a_{i^{*}}=p$. If $\#\left\{i \in N \mid a_{i}=p\right\}>\#\left\{i \in N \mid a_{i}=q\right\}$ then project $p$ is successful and player $i^{*}$ has no incentives to switch to $q$. In addition, all peripherals moderate players who choose project $q$ (i.e. all $j \in N_{m}$ such that $a_{j}=q$ ) have incentives to choose project $p$ instead of $q$. If $\#\left\{i \in N \mid a_{i}=p\right\} \leq \#\left\{i \in N \mid a_{i}=q\right\}$ then project $q$ is successful and player $i^{*}$ has incentives to switch to $q$. After player $i^{*}$ has switched to $q$, all peripherals moderate players who choose project $p$ (i.e. all $j \in N_{m}$ such that $a_{j}=p$ ) have incentives to choose project $q$ instead of $p$. Hence, $h(a) \neq \emptyset$ and $a \notin \widehat{Z}(g, t)$. (ib) Take any $a \in\left\{a \in A \mid a_{i}=a_{j}\right.$ for all $\left.i, j \in N_{m}\right\}$ and suppose (without loss of generality) that $a_{i}=p$ for all $i \in N_{m}$. Since player $i^{*}$ is myopic she has no incentives to switch to project $q, U_{i^{*}}\left(a_{i^{*}}^{\prime}=q, a_{-i^{*}}^{\prime}=a_{-i^{*}}\right)<U_{i^{*}}\left(a_{i^{*}}=p, a_{-i^{*}}\right)$. Since player $i^{*}$ chooses $a_{i^{*}}=p$, all peripherals (farsighted or myopic) moderate players have no 
incentives to choose project $q$ instead of project $p$. Hence, $h(a)=\emptyset$ and $a \in \widehat{Z}(g, t)$. From (ia) and (ib) we have $\widehat{Z}(g, t)=\left\{a \in A \mid a_{i}=a_{j}\right.$ for all $\left.i, j \in N_{m}\right\}$ if $i^{*} \in M$.

(ii) Suppose $i^{*} \in F, \# F+\# N_{q} \geq t^{q}$ and $\# N_{q}>\# N_{p}$. (iia) Take any $a \notin\{a \in A \mid$ $a_{i}=q$ for all $\left.i \in N_{m}\right\}$. If $a_{i^{*}}=q$, then all peripheral farsighted players have incentives to choose the same project $q$ as player $i^{*}$. Indeed, once they have chosen $q$, this project is successful for sure $\left(\# F+\# N_{q} \geq t^{q}\right)$. Afterwards, all peripheral myopic players who were choosing project $p$ have incentives to switch to project $q$. If $a_{i^{*}}=p$, then player $i^{*}$ who is farsighted has incentives to switch to $q$ anticipating that all peripheral farsighted players will have incentives to choose the same project $q$ as player $i^{*}$, followed by all peripheral myopic players who will now have incentives to choose project $q$. Hence, $h(a) \neq \emptyset$ if $a \notin\left\{a \in A \mid a_{i}=q\right.$ for all $\left.i \in N_{m}\right\}$. (iib) Take any $a \in\left\{a \in A \mid a_{i}=q\right.$ for all $\left.i \in N_{m}\right\}$. Obviously, $h(a)=\emptyset$ since $U_{i}(a) \geq U_{i}\left(a^{\prime}\right)$ for all $i \in N_{m}$, for all $a^{\prime} \in A$. Hence, $a \in \widehat{Z}(g, t)$. From (iia) and (iib) we have $\widehat{Z}(g, t)=\left\{a \in A \mid a_{i}=q\right.$ for all $\left.i \in N_{m}\right\}$.

(iii) Suppose $i^{*} \in N_{q}$ and $\# F+\# N_{q} \geq t^{q}$. (iiia) Take any $a \notin\left\{a \in A \mid a_{i}=q\right.$ for all $\left.i \in N_{m}\right\}$. All peripheral farsighted players have incentives to choose the same project $q$ as the stubborn player $i^{*}$. Indeed, once they have chosen $q$, this project is successful for sure $\left(\# F+\# N_{q} \geq t^{q}\right)$. Afterwards, all peripheral myopic players who were choosing project $p$ have incentives to switch to project $q$. Hence, $h(a) \neq \emptyset$ if $a \notin\left\{a \in A \mid a_{i}=q\right.$ for all $\left.i \in N_{m}\right\}$. (iiib) Take any $a \in\left\{a \in A \mid a_{i}=q\right.$ for all $\left.i \in N_{m}\right\}$. Obviously, $h(a)=\emptyset$ since $U_{i}(a) \geq U_{i}\left(a^{\prime}\right)$ for all $i \in N_{m}$, for all $a^{\prime} \in A$. Hence, $a \in \widehat{Z}(g, t)$. From (iiia) and (iiib) we have $\widehat{Z}(g, t)=\left\{a \in A \mid a_{i}=q\right.$ for all $\left.i \in N_{m}\right\}$.

Proof of Proposition 9. Take the line network $\{12,23,34,45, \ldots\}$. $(\Rightarrow)$ Let us assume that player $i \in\{2, \ldots, n-1\}$ is myopic while players $j \neq i \in\{2, \ldots, n-1\}$ are farsighted. Obviously the strategy profiles in which all players choose the same action are stable. We show that no other strategy profile can be stable:

(i) Let us assume that two farsighted neighbors $j, k$ (with $k=j+1$ ) choose different projects and all $l<j$ choose project $a_{j}$ and all $l>k$ choose project $a_{k}$. Without loss of generality we assume that $i>k$, i.e. $a_{i}=a_{k}$. Then player $j$ has an incentive to change her decision and to select project $a_{k}$. If player $j$ changes her project, then player $l=j-1$ changes her project, because she knows that her neighbor $m=j-2$ will change afterwards. Player $j-x$ changes, because the farsighted player 2 who is the neighbor of the loose end knows that if she changes her decision the loose end will follow.

(ii) Let us assume without loss of generality that player $i$ chooses project $q$ and all players located to her left do the same, i.e. $a_{j}=a_{i}=q$ for all $j \in N$ with $j<i$ while all other players choose $p$. In this case the neighbor of player $i$ who chooses a different project than $i$, namely player $i+1$ (with $a_{i+1}=p$ ) has an incentive 
to deviate. Player $i+1$ changes because all other players $i+2$ to $n$ will change afterwards. These players change, because they anticipate that player $n$ (the looseend) will change as well.

So under these assumptions there can be no other stable strategy profile.

$(\Leftarrow)\left(t^{p}=t^{q}=1\right)$ We assume there are two myopic players $i, j \in\{2, \ldots, n-1\}$.

(i) Assume $i$ and $j$ are neighbors, i.e. $j=i+1$. Then there exists a stable strategy profile in which all players $k \leq i$ choose project $q$ and all players $l \geq j=i+1$ choose project $p$. In that case the farsighted players 2 to $i-1$ and player 1 (who can be farsighted or myopic, it does not matter) choose $q$ and the farsighted players $j+1$ to $n-1$ and player $n$ choose project $p$. This implies that neither of the players 1 to $i-1$ to $j+1$ to $n$ has any incentive to deviate. The two myopic players have also no incentive to change, because their utility after changing would be the same as before.

(ii) Assume $i$ and $j$ are not neighbors, without loss of generality let $i<j$. We show that the following strategy profile is stable: $a_{k}=q$ for all $k<j$ and $a_{k}=p$ for all $k \geq j$. In this strategy profile there are some farsighted players between $i$ and $j$ who choose project $q$. The myopic player $j$ has no incentive to deviate to project $q$, because one of her neighbors chooses $q$ and the other chooses $p$. The farsighted player $j-1$ would choose project $p$ only if $j-2$ chooses project $p$ as well. Player $j-2$ would deviate to project $p$ only if $j-3$ deviates to $p$ as well, continuing this argument leads to the problem that $i+1$ only chooses project $p$ if $i$ deviates to $p$. Since $i$ is myopic she does not deviate, because even if $i+1$ choose $p$, she still gets the same utility from the projects $q$ and $p$. So the strategy profile described is stable.

Proof of Proposition 10. Take $S_{q}^{l}\left(g^{c}, a\right)=\left\{i_{1}, i_{2}, \ldots, i_{K}\right\}$ where $K=\# S_{q}^{l}\left(g^{c}, a\right)$. (i) Since $i_{k} \in N_{i_{k+1}}\left(g^{c}\right)$ for all $i_{k}, i_{k+1} \in S_{q}^{l}\left(g^{c}, a\right)$, the fragment is a line. Hence, the proof of Proposition 9 applies here. (ii) Players $i_{1}$ and $i_{K}$ have one neighbor choosing project $q$ and one neighbor choosing project $p$. By adding a new link $i_{1} j$ where $a_{j}=p$, we have $U_{i_{1}}\left(p, a_{-i_{1}}\right)>U_{i_{1}}\left(q, a_{-i_{1}}\right)$ and then player $i_{1}$ switches to project $p$. Analogously for player $i_{K}$. Now players $i_{2}$ and $i_{K-1}$ have one neighbor choosing project $q$ and one neighbor choosing project $p$. We next proceed to add links among $i_{2}$ and $i_{K-1}$ with two other players choosing $p$. We continue adding links between players in the fragment (with neighbors choosing $q$ and $p$ ) and players choosing $p$ until there is a unique player $i_{k}$ in the fragment who has both neighbors choosing project $p$, and so she has incentives to choose $p$. Therefore, we need to add $\# S_{q}^{l}\left(g^{c}, a\right)-1$ links to obtain full coordination on project $p$. (iii) We repeat the previous case (ii) until there are two players $i_{k}, i_{k+1}$ in the fragment 
who have one neighbor choosing project $q$ and one neighbor choosing project $p$. Suppose that player $i_{k}$ is farsighted, $\# F=1$, she will switch to project $p$, anticipating that the myopic player $i_{k+1}$ will join project $p$ as well. Therefore, with $\# F=1$ farsighted player and $\# L=\# S_{q}^{l}\left(g^{c}, a\right)-1-\# F=\# S_{q}^{l}\left(g^{c}, a\right)-2$ links we obtain the full coordination in the fragment. Following the same algorithm, if we increase the number of farsighted players $\left(\# F<\# S_{q}^{l}\left(g^{c}, a\right)-1\right)$ in the fragment, we could reduce the number of links needed for full coordination within the fragment.

Proof of Proposition 11. Take $S_{q}^{l}\left(g^{c}, a\right)=\left\{i_{1}, i_{2}, ., i_{k}, . ., i_{K}\right\}$ where $K=\# S_{q}^{l}\left(g^{c}, a\right)$. Remember that player $i_{k} \in N_{q}$ is stubborn for project $q$, and so $a_{i_{k}}=q$. (i) We start to add links to the players at the extreme of the fragment, $i_{1}$ and $i_{K}$, with two other players choosing $p$. By doing so, players $i_{1}$ and $i_{K}$ will now have incentives to choose $p$. We continue this process of adding links between the new players at the extreme of the fragment choosing still $q$ and players choosing $p$ until we end up with player $i_{k}$. By adding $\# S_{q}^{l}\left(g^{c}, a\right)-1$ links, $\# S_{q}^{l}\left(g^{c}, a\right)-1$ moderate agents have now a new neighbor choosing project $p$, and thus have incentives to switch to project $p$. (ii) If $S_{q}^{l}\left(g^{c}, a^{\prime}\right)=\left\{i_{k-1}, i_{k}, i_{k+1}\right\}$ with $i_{k}$ as stubborn for $q$, only adding links (as in (i)) works in this case. Otherwise, we start by adding two initializing links between $i_{k-1}$ and $i_{k+1}$ with two moderates choosing $p$. Then, we go back to the extreme players in the fragment $i_{1}$ and $i_{K}$. Gradually adding one link to each player in the extreme of the fragment, $S_{q}^{l}\left(g^{c}, a\right)$, with a moderate choosing $p$ makes these players in the fragment choosing project $p$. Let us assume that $i_{k-2}$ and $i_{k+2}$ are farsighted, then as soon as $i_{k-3}$ adopts project $p, i_{k-2}$ switches to $p$ as well, because she knows that $i_{k-1}$ now has two neighbors choosing $p$ and one neighbor $i_{k}$ choosing $q$. Obviously, $i_{k-1}$ joins $p$, thereby increasing his own utility and the utility of his farsighted neighbor $i_{k-2}$. Analogously for $i_{k+2}$ and $i_{k+1}$. Therefore, we have $\# F=2$ and $\# L=\# S_{q}^{l}\left(g^{c}, a\right)-1-\# F$ links to moderate players choosing $p$. Increasing the number of farsighted players will decrease the number of links to moderate

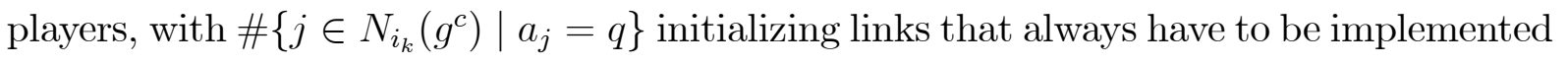
first, so $\# F \leq \# S_{q}^{l}\left(g^{c}, a\right)-1-\#\left\{j \in N_{i_{k}}\left(g^{c}\right) \mid a_{j}=q\right\}$.

\section{References}

[1] Allouch, N., 2015. On the private provision of public goods on networks. Journal of Economic Theory 157, 527-552.

[2] Bramoullé, Y., 2007. Anti-coordination and social interactions. Games and Economic Behavior 58, 30-49. 
[3] Bramoullé, Y., D. Lopez-Pintado, S. Goyal and F. Vega-Redondo, 2004. Network formation and anti-coordination games. International Journal of Game Theory 33, $1-19$.

[4] Bramoullé, Y. and R. Kranton, 2007. Public goods in networks. Journal of Economic Theory 135, 478-494.

[5] Chwe, M.S., 1994. Farsighted coalitional stability. Journal of Economic Theory 63, 299-325.

[6] Dutta, B., S. Ghosal and D. Ray, 2005. Farsighted network formation. Journal of Economic Theory 122, 143-164.

[7] Goyal, S., 2007. Connections: an introduction to the economics of networks. Princeton University Press: Princeton, NJ, USA.

[8] Goyal, S. and F. Vega-Redondo, 2005. Network formation and social coordination. Games and Economic Behavior 50, 178-207.

[9] Herings, P.J.J., A. Mauleon and V. Vannetelbosch, 2009. Farsightedly stable networks. Games and Economic Behavior 67, 526-541.

[10] Herings, P.J.J., A. Mauleon and V. Vannetelbosch, 2017. Matching with myopic and farsighted players. CORE Discussion Paper 2017-14, University of Louvain.

[11] Herings, P.J.J., A. Mauleon and V. Vannetelbosch, 2019. Stability of networks under horizon-K farsightedness. Economic Theory 68, 177-201.

[12] Jackson, M.O., 2008. Social and economic networks. Princeton University Press: Princeton, NJ, USA.

[13] Jackson, M. O. and E. Storms, 2019. Behavioral communities and the atomic structure of networks. Mimeo, Stanford University, CA, USA.

[14] Jackson, M. O. and A. Watts, 2002a. On the formation of interaction networks in social coordination games. Games and Economic Behavior 41, 265-291.

[15] Jackson, M.O. and A. Watts, 2002b. The evolution of social and economic networks. Journal of Economic Theory 106, 265-295.

[16] Kirchsteiger, G., M. Mantovani, A. Mauleon and V. Vannetelbosch, 2016. Limited farsightedness in network formation. Journal of Economic Behavior and Organization $128,97-120$.

[17] Leister, C. M., Y. Zenou and J. Zhou, 2020. Coordination on networks. Mimeo, Monash University, Australia. 
[18] Mauleon, A. and V. Vannetelbosch, 2016. Network formation games. In The Oxford Handbook of The Economics of Networks (Y. Bramoullé, A. Galeotti and B.W. Rogers, eds.), Oxford University Press, UK.

[19] Page, F.H., Jr. and M. Wooders, 2009. Strategic basins of attraction, the path dominance core, and network formation games. Games and Economic Behavior 66, 462487.

[20] Ray, D. and R. Vohra, 2015. The farsighted stable set. Econometrica 83, 977-1011.

[21] Ray, D. and R. Vohra, 2019. Maximality in the farsighted stable set. Econometrica 87, 1763-1779.

[22] Sakovics, J. and J. Steiner, 2012. Who matters in coordination problems? The American Economic Review 102, 3439-3461. 